\title{
Graphene-Based Nanomaterials for Theranostic Applications
}

\author{
Shounak Roy and Amit Jaiswal* \\ School of Basic Sciences, Indian Institute of Technology Mandi \\ Kamand, Mandi 175005, Himachal Pradesh, India \\ BioX Centre, Indian Institute of Technology Mandi \\ Kamand, Mandi 175005, Himachal Pradesh, India \\ *j.amit@iitmandi.ac.in
}

Published 15 January 2018

\begin{abstract}
Graphene and graphene-based nanomaterials such as graphene oxide (GO), reduced graphene oxide (rGO) and graphene quantum dots (GQDs) have gained a lot of attention from diverse scientific fields for applications in sensing, catalysis, nanoelectronics, material engineering, energy storage and biomedicine due to its unique structural, optical, electrical and mechanical properties. Graphene-based nanomaterials emerge as a novel class of nanomedicine for cancer therapy for several reasons. Firstly, its structural properties like high surface area and aromaticity enables easy loading of hydrophobic drugs. Secondly, presence of oxygen containing functional groups improve its physiological stability and also act as site for biofunctionalization. Thirdly, its optical absorption in the NIR region enable them to act as photoagents for photothermal and photodynamic therapies of cancer, both in vitro and in vivo. Finally, its intrinsic fluorescence property helps in bioimaging of cancer cells. Overall, graphene-based nanomaterials can act as agents for developing multifunctional theranostic platforms for carrying out more efficient detection and treatment of cancers. This review provides a detailed summary of the different applications of graphene-based nanomaterials in drug delivery, nucleic acid delivery, phototherapy, bioimaging and theranostics.
\end{abstract}

Keywords: Graphene; graphene oxide; reduced graphene oxide, drug delivery; nucleic acid delivery; phototherapy; bioimaging; theranostics.

\section{Introduction}

With its discovery in the year 2002 followed by a Nobel prize in Physics in 2010, graphene has attracted a lot of attention from diverse scientific fields for applications in sensing, catalysis, nanoelectronics, material engineering, energy storage and biomedicine. The reason behind this diverse set of applications is its unique structural,

*Corresponding author.

This is an Open Access article published by World Scientific Publishing Company. It is distributed under the terms of the Creative Commons Attribution 4.0 (CC-BY) License. Further distribution of this work is permitted, provided the original work is properly cited. 
optical, electrical, and mechanical properties that have been found to be superior than other widely explored carbon and plasmonic nanomaterials. ${ }^{1-4}$ Graphene is a two-dimensional (2D) nanomaterial having a monoatomic layer of $s p^{2}$ hybridized carbon atoms organized in the form of a honeycomb lattice. ${ }^{5}$ Synthesis of graphene oxide (GO) and reduced graphene oxide (rGO) have been made possible with the development of new synthesis methods that have further enhanced interest in this material specifically in the biomedical field due to the incorporation of new features and novel properties in these derivatives (Fig. 1) ${ }^{5-7}$ The ultra-thin nanosheets of graphene and its derivatives like GO and rGO provide very high surface area for efficient loading of therapeutic cargoes like drugs, nucleic acids, fluorescent probes, biomolecules and cells. Additionally, these materials also provide a distribution of delocalized $\pi$ electrons on the surface giving it an aromatic character, that help in increasing the loading and binding efficiency of graphene-based nanomaterials with other molecules of therapeutic interest through hydrophobic interactions and $\pi-\pi$ stacking. ${ }^{5}$ The synthesis procedures of GO introduce wide variety of oxygen containing functional groups like carboxyl, hydroxyl, carbonyl, ethoxy, epoxy, etc., on both planes of GO sheets, thereby increasing its stability in aqueous solutions. This also provides easy functionalization and derivatization of these materials through both covalent and non-covalent modifications with different biocompatible polymers like PEG, chitosan and conjugation with targeting moieties like peptides and antibodies, to develop biofunctionalized nanocomposite systems with improved biological properties. ${ }^{5,8,9}$ Apart from these surface properties, GO and rGO also possess exciting intrinsic optical properties like photoluminescence and increased absorbance in the near-infrared region (NIR) of electromagnetic spectrum, that make them excellent photo-responsive agents for cancer therapies like photothermal therapy (PTT) and photodynamic therapy (PDT). ${ }^{5}$ Similar to plasmonic nanomaterials, GO and rGO show photothermal conversion properties in response to NIR irradiation that has been widely explored as a therapeutic strategy for treating cancers. In this context, rGO has been found to be much more efficient than GO because of the

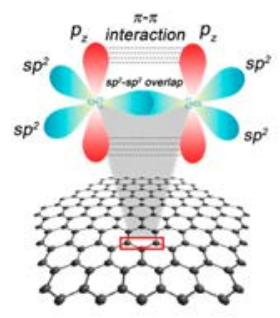

Graphene

(a)

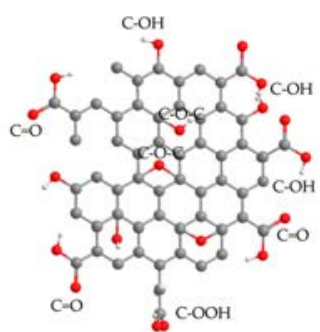

Graphene oxide

(b)

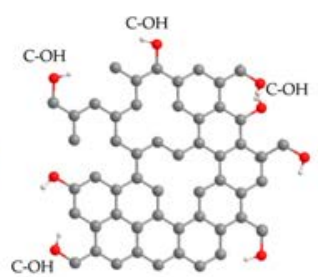

Reduced graphene oxide

(c)

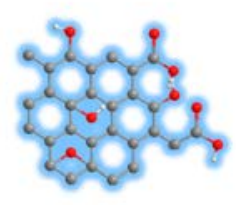

Graphene-based quantum dot

(d)

Fig. 1. Structures of graphene-based materials show (a) the pristine graphene (pure-arranged carbon atoms) with $s p^{2}$-hybridized carbon atoms, and the chemically modified graphene, including (b) GO; (c) rGO and (d) graphene quantum dot (GQD). Reproduced with permission from Ref. 7. 
presence of more defects and greater aromaticity in its structure that are introduced into it during reduction of GO. ${ }^{5}$ Owing to these excellent physicochemical and biocompatible properties, graphene-based nanomaterials have found a wide variety of applications in the field of biomedicine, mostly in cancer research, ranging from serving as nanocarriers and photoagents for drug/gene delivery to cancer cells and for photothermal and photodynamic therapies of cancer, both in vitro and in vivo, till its use as bioimaging agents for developing multifunctional theranostic platforms for carrying out more efficient detection and treatment of cancers. ${ }^{10}$

In this review, we provide a detailed and systematic description of the journey that graphene-based nanomaterials have undertaken in the field of biomedical sciences in the last decade by discussing the different applications in drug delivery, gene delivery, phototherapy, bioimaging and theranostics. The main objective of this review is to make the readers understand the evolution of graphene as a nanomedicine over the years and appreciate the immense potential that this nanomaterial holds for the future.

\section{Graphene-Based Nanomaterials for Drug Delivery}

In the recent years, graphene-based nanomaterials such as GO and rGO have emerged as excellent nanocarriers for drug delivery owing to their structural and surface properties that make them very suitable for biomedical applications. ${ }^{10,11}$ The monolayer 2D structure of GO and rGO provide ultrahigh specific surface area and delocalized $\pi$ electrons on the surface that allow highly efficient loading of hydrophobic anti-cancer drugs through hydrophobic interactions and $\pi-\pi$ stacking, and also provide different oxygen containing functional groups for easy biofunctionalization. ${ }^{12-15}$ In this section, we discuss the different drug delivery applications of graphene-based nanomaterials under two broad categories - (A) Direct drug delivery systems and (B) Stimulus responsive drug delivery systems, to understand the different strategies by which graphene and its derivatives have been exploited for improving cancer chemotherapy. Direct drug delivery systems involve delivery of drugs to a cell or tissue either in a targeted or non-targeted manner without any control on the delivery mechanism. On the other hand, stimulus responsive drug delivery systems provide a controlled and systematic release of drugs from the nanocarrier in response to a specific stimulus, which can be both internal such as $\mathrm{pH}$, redox, etc., as well as external such as temperature, light, magnetic and electrical field, etc. Table 1 summarizes the different graphene-based drug delivery systems developed so far.

\subsection{Direct drug delivery systems}

\subsubsection{Non-targeted drug delivery}

Delivery of anti-cancer drugs using GO as a nanocarrier was reported for the first time by Liu et al. in 2008. ${ }^{16}$ They used PEGylated GO to load a hydrophobic 
Table 1. Summary of graphene-based nanomaterials for drug delivery.

\begin{tabular}{|c|c|c|c|}
\hline Nanocomposite & Drug & Target & Reference \\
\hline \multirow[t]{3}{*}{ PEG-GO } & SN38 & HCT-116 cells & 16 \\
\hline & DOX & & 17 \\
\hline & DOX \& CPT & MCF-7 cells & 18 \\
\hline nGr-PLA-PEG & PTX & U-138 glioblastoma cells & 19 \\
\hline PEG-GO & PTX & A549 \& MCF-7 cells & 20 \\
\hline PLA-PEG-GO & PTX & A549 cells & 21 \\
\hline PDA-GO-FA & DOX & & 22 \\
\hline HA-GO & DOX & $\begin{array}{l}\text { KB epidermal carcinoma } \\
\text { cells and tumors }\end{array}$ & 23 \\
\hline HA-GO & DOX & HeLa tumors & 24 \\
\hline Tf-PEG-GO & DOX & Glioma & 25 \\
\hline CTX-GO & DOX & Glioma & 26 \\
\hline GONR-PEG-DSPE & Lucanthone & $\begin{array}{l}\text { EGFR positive glioblasto- } \\
\text { ma multiforme cells }\end{array}$ & 29 \\
\hline GO/PVA hydrogel & Vitamin B12 & & 30 \\
\hline GO-CS-FA & DOX & & 31 \\
\hline $\begin{array}{l}\text { Pluronic F38, Tween } 80 \\
\text { and maltodextrin } \\
\text { functionalized GO }\end{array}$ & Ellagic acid & MCF-7 \& HT29 cells & 32 \\
\hline GO & $\mathrm{ADR}$ & MCF-7/ADR & 33 \\
\hline $\begin{array}{l}\text { Lipid monolayer membrane } \\
\text { functionalized graphene } \\
\text { sheets }\end{array}$ & DOX & & 34 \\
\hline GN-CNT-Fe ${ }_{3} \mathrm{O}_{4}$ & 5 -FU & HepG2 cells & 35 \\
\hline HA-GO & DOX & $\begin{array}{l}\mathrm{H} 22 \text { hepatic carcinoma } \\
\text { tumors }\end{array}$ & 36 \\
\hline Albumin-GO & PTX & & 37 \\
\hline $\begin{array}{l}\text { rGO-C6H4-CO-NH-PEI-NH- } \\
\text { CO-CD-Biotin }\end{array}$ & DOX & HepG2 cells & 38 \\
\hline GO-Abs/PEI/PAH-Cit & DOX & U87 MG \& MCF-7 cells & 39 \\
\hline NGO-SS-mPEG & DOX & HeLa cells & 40 \\
\hline PEG-GO & DOX & A549 cells & 41 \\
\hline PEG-BPEI-rGO & DOX & PC-3 cells & 42 \\
\hline Graphene $/ \mathrm{Fe}_{3} \mathrm{O}_{4}$ composites & DOX & & 43 \\
\hline $\mathrm{Fe}_{3} \mathrm{O}_{4} / \mathrm{GNS}$ & Rhodamine B & & 44 \\
\hline rGO/PVA hydrogel & Lidocaine hydrochloride & & 45 \\
\hline
\end{tabular}

anti-cancer drug SN38 onto the surfaces of GO through $\pi-\pi$ stacking and hydrophobic interactions. This SN38 loaded GO-PEG nanocarrier was then used to deliver the drug to HCT-116 colon cancer cells in vitro. It was observed that the nanocarriers could efficiently deliver the drug into the cancer cells and demonstrated 2-3 fold improvement in the therapeutic efficiency as compared to the water soluble prodrug of SN38, irinotecan. In the same year, Yang et al. also showed highly efficient loading and delivery of another very commonly used hydrophobic cancer drug, doxorubicin using GO as a nanocarrier. ${ }^{17}$ To further improve the therapeutic efficiency, Zhang et al. loaded both doxorubicin and camptothecin onto GO sheets and demonstrated efficient killing of MCF-7 breast cancer cells by combining the efficacies of both drugs into a single platform. ${ }^{18}$ Moore et al. demonstrated drug delivery to glioblastomas 
using biodegradable polylactic acid (PLA) coated nanographene (nGr) functionalized with PEG (nGr-PLA-PEG) as a biocompatible drug delivery system. ${ }^{19}$ nGr-PLA-PEGs could encapsulate the anticancer drug Paclitaxel (PTX) at a high loading efficiency and showed controlled release of the drug over a period of 19 days. PTX loaded nGr-PLA-PEGs exhibited $20 \%$ killing of U-138 glioblastoma cells in vitro and in vivo biodistribution studies revealed accumulation of $\mathrm{n} \mathrm{Gr}$ in intracranial U-138 glioblastoma xenografts and organs of the reticuloendothelial system. In a recent study, PEGylated GO was used as a nanocarrier for the delivery of PTX to A549 human lung cancer cells and MCF-7 breast cancer cells in vitro. ${ }^{20}$ PTX was first attached to a 6 -armed PEG which was then covalently conjugated to GO sheets. The GO-PEG-PTX nanocomposites showed efficient cellular uptake and greater cytotoxicity against the cancer cells as compared to free PTX (Fig. 2(i)). PLA-PEG stabilized GO nanocomposites were developed and used as a drug delivery vehicle by Angelopoulou et al. for delivering PTX to A549 human lung cancer cells in culture. $^{21}$ The nanocomposites showed efficient loading and sustained drug release properties alongwith significant cellular uptake and cytotoxicity against the cancer cells.

\subsubsection{Targeted drug delivery}

Cancer cell specific targeted drug delivery systems have also been developed using graphene-based nanomaterials for further increasing the therapeutic efficiency by selectively delivering the drugs into cancer cells, thereby minimizing the dose of the therapeutic load and also avoiding unnecessary side-effects to normal cells. Lin et al. reported the synthesis of folic acid decorated, polydopamine capped GO nanocomposites by a one-pot method and demonstrated targeted delivery of doxorubicin to cancer cells in vitro with high therapeutic efficiency. ${ }^{22}$ Cholesteryl hyaluronic acid (HA) conjugated GO loaded with doxorubicin was developed by Miao et al. for targeted delivery of doxorubicin to KB epidermal carcinoma cells both in in vitro and in vivo. ${ }^{23}$ In vitro studies showed that the targeted nanocomposite induced $40.3 \%$ increase in cell death as compared to the non-targeted one. Similarly, subcutaneous injection of the targeted nanocomposites in tumor bearing athymic mice resulted in $14.1 \%$ decrease in the tumor volume within 24 days. In another study, HA was linked to $\mathrm{GO}$ via an adipic acid dihydrazide $(\mathrm{ADH})$ linkage to form HA tagged GO-based targeted drug delivery system that was used for delivering doxorubicin to mice with subcutaneous HeLa tumors. ${ }^{24}$ Within 16 days, the targeted nanocomposites showed $12 \%$ and $17 \%$ greater tumor inhibition rates as compared to free doxorubicin and non-targeted nanocomposites, respectively. In addition to FA and HA, transferrin (Tf) protein is also used as a targeting agent. Liu et al. synthesized Tf conjugated PEGylated nanographene oxide composites loaded with doxorubicin (Tf-PEG-GODox) for targeted delivery of drug to glioma cells. ${ }^{25}$ The nanocomposites showed a drug loading ratio of up to $115.4 \%$ and demonstrated significantly higher cytotoxicity against gliomas as compared to free drug and PEG-GO-Dox, both in in vitro 


\section{(i) Non-Targeted DDS}

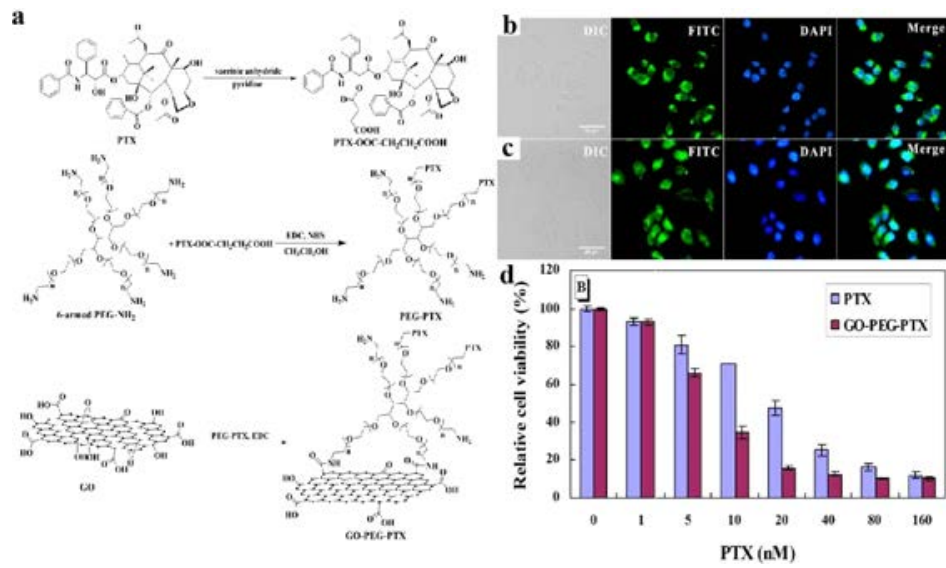

(ii) Targeted DDS

a
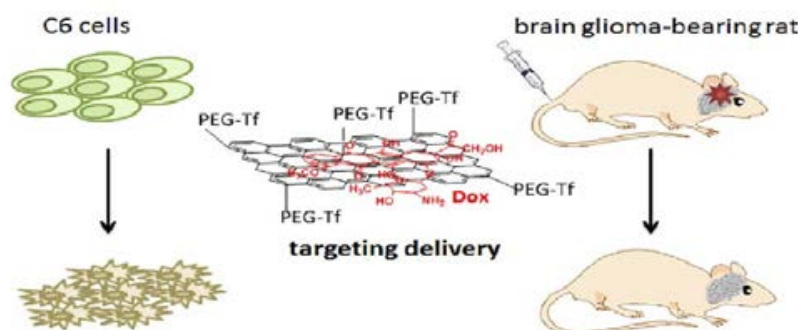

targeting delivery

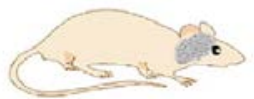

b
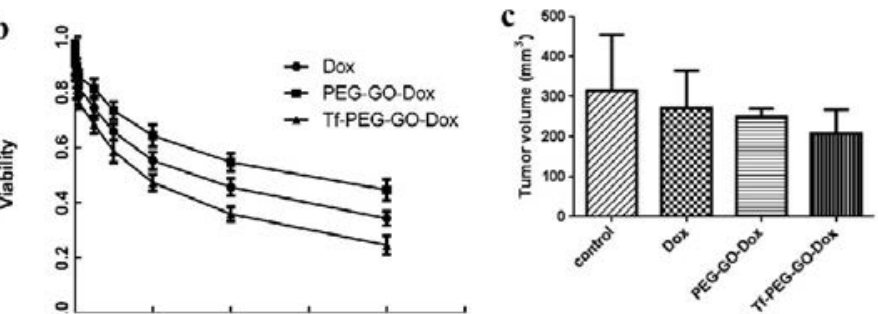

Fig. 2. Graphene-based nanomaterials for drug delivery. (i) Non-targeted drug delivery system (a) Preparation of GO-PEG-PTX Nanoscale Drug Delivery System. Differential interference contrast (DIC), FITC, DAPI, and merge (FITC + DAPI) images of A549, (b) and MCF-7, (c) cells incubated with GO-PEG-FITC for $3 \mathrm{~h}$; scale bar $25 \mu \mathrm{m}$, (d) Relative cell viability of MCF-7 cells after treatment with different concentrations of PTX and GO-PEG-PTX for $72 \mathrm{~h}$. Reproduced with permission from Ref. 20. Copyright 2014 American Chemical Society. (ii) Targeted drug delivery system (a) Schematic representation of targeted delivery of Tf-PEG-GO-Dox to cells in vitro and in vivo. (b) C6 viability-concentration curves for Dox, PEG-GO-Dox, and Tf-PEG-GO-Dox. (c) Glioma volume in different therapeutic groups on the 14th day. Reproduced with permission from Ref. 25. Copyright 2013 American Chemical Society. (iii) Internal-stimulus responsive drug delivery system. Schematic diagram showing antitumor activity of redox-sensitive DXR-loaded NGO-SS-mPEG Reproduced with permission from Ref. 40. Copyright 2012 Wiley-VCH Verlag GmbH \& Co. KGaA, Weinheim. (iv) External-stimulus responsive drug delivery system. Schematic representation of photothermally triggered cytosolic drug delivery via endosomal disruption using PEG-BPEI-rGO. Reproduced with permission from Ref. 42. Copyright 2013 American Chemical Society. 
(iii) Internal Stimuli Responsive DDS

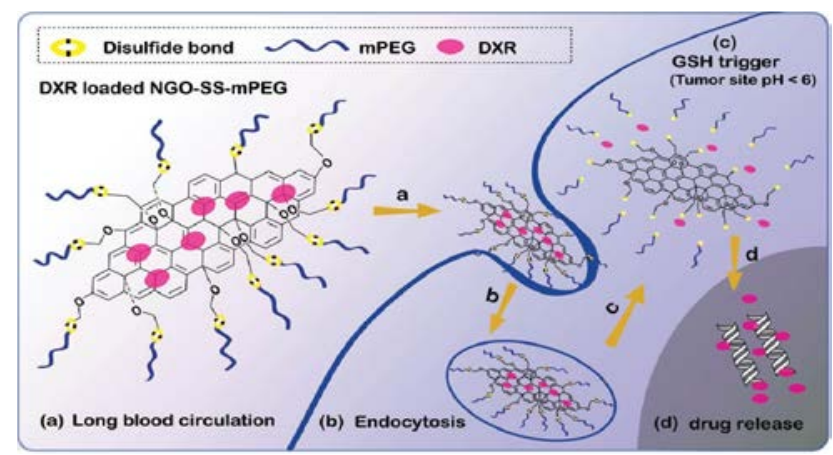

(iv) External Stimuli Responsive DDS

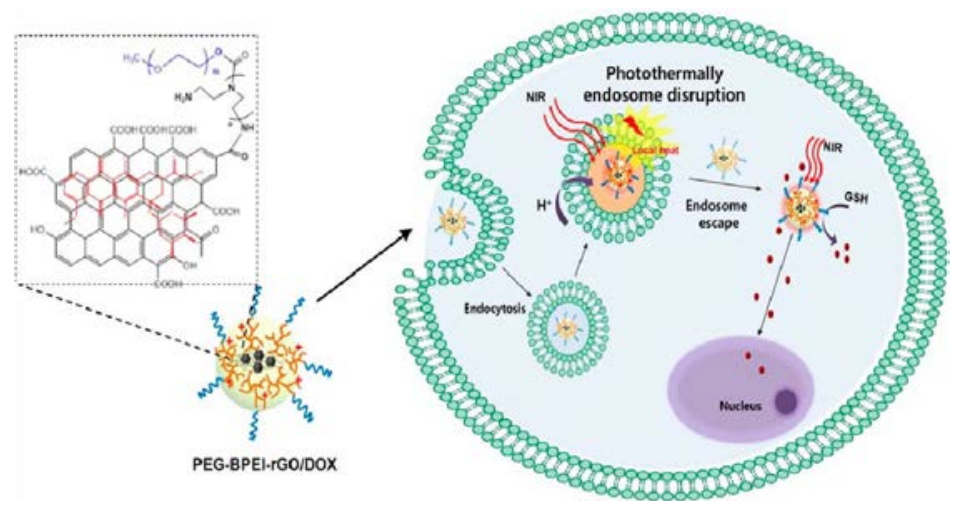

Fig. 2. (Continued)

and in vivo (Fig. 2(ii)). Another glioma targeted drug delivery system was developed by Wang et al., where they conjugated GO with chlorotoxin (CTX-GO) as the targeting agent for selective delivery of doxorubicin to glioma cells. ${ }^{26}$ Chlorotoxin is a toxin derived from Leiurus quinquestriatus having high affinity for matrix-metalloprotease-2 proteins that are known to be overexpressed in gliomas. CTX-GO loaded with doxorubicin showed higher accumulation of drug inside gliomas and caused greater cytotoxicity against glioma cells as compared to free drug and GO-Dox. A new type of graphene derivative referred to as graphene oxide nanoribbons (GONR) was synthesized by Chowdhury et al. by using carbon nanotubes (CNT) as the precursor material. ${ }^{27}$ These GONRs upon functionalization with PEG-DSPE (1, 2-distearoyl-sn-glycero-3 phosphoethanolamine) (GONR-PEG-DSPE) showed cancer cell targeted cytotoxicity without the use of a targeting agent. The targeting specificity was found towards cells overexpressing EGFR and this property of GONRs was exploited by the group to selectively deliver lucanthone 
(apurinic endonuclease-1 (APE-1) inhibitor) to EGFR over-expressing glioblastoma multiforme cells in culture. ${ }^{28,29}$ Lucathone-GONR nanocomposites showed significant cytotoxicity against glioblastoma multiforme cells but demonstrated no toxicity to EGFR negative glial precursor cells, thereby showing the specificity of GONRs as a novel drug delivery system.

\subsection{Stimuli-responsive drug delivery systems}

Stimulus-responsive drug delivery systems allow for a controlled release of drugs at a particular site only in the presence of a specific stimulus, that directs the nanocarrier system to release its therapeutic payload. Controlled release of drugs improves the therapeutic efficiency of the system by minimizing the chances of premature and burst release of drugs in the circulation. Stimulus can be either internal or external. Internal stimulus refers to the changes in the intracellular environment such as $\mathrm{pH}$, redox, etc., that can trigger release of drugs from nanocarriers specifically designed to respond to such stimuli, or it can respond to external stimuli such as light, temperature, magnetic and electrical field.

\subsubsection{Internal stimuli responsive systems}

Several graphene-based drug delivery systems have been developed in the recent years that are responsive to internal stimulus like $\mathrm{pH}$ and redox. Cancer cells and tumor microenvironments are known to have an acidic $\mathrm{pH}$ and high concentrations of redox enzymes like GSH as compared to surrounding healthy cells and tissues. So, these two intracellular stimuli have been exploited to efficiently modulate and control the release of drugs from nanocarriers specifically inside the tumor cells and tissues. Bai et al. reported a $\mathrm{pH}$-sensitive composite hydrogel consisting of GO and poly (vinyl alcohol) (GO/PVA) that showed pH-responsive gel-sol transition properties, thereby allowing efficient loading and release of drugs at different $\mathrm{pH}$ conditions. ${ }^{30}$ Depan et al. developed a doxorubicin loaded GO-chitosan-FA nanocomposite (DOX-GO-CH-FA) and studied its drug release properties in response to different $\mathrm{pH}$ values. ${ }^{31}$ It was observed that the nanocomposites showed higher and faster release of DOX at an acidic $\mathrm{pH}$ than physiological $\mathrm{pH}$, and also showed morphological transition from non-porous to porous structure after drug release. Similarly, a biocompatible polymer pluronic F38, Tween80 and maltodextrin functionalized GO nanocomposite was prepared by Kakran et al. which showed good loading of a poorly water soluble anti-cancer drug ellagic acid and increased release profiles at acidic $\mathrm{pH} .^{32}$ In an interesting study by Wu et al., GO was used as carrier for adriamycin (ADR) to overcome ADR resistance in MCF-7/ADR breast cancer cells in vitro. ${ }^{33}$ GO showed a high ADR loading efficiency of around $93.6 \%$ and demonstrated $\mathrm{pH}$-dependent release of $\mathrm{ADR}$. The in vitro studies revealed that $\mathrm{GO}$ could efficiently deliver the drug into MCF-7/ADR cells and have significantly higher cytotoxicity against the drug resistant cancer cells in comparison to free ADR. Lipid monolayer membrane functionalized graphene sheets were developed by 
Liu et al. as highly efficient physiologically stable $\mathrm{pH}$ responsive drug delivery system, that showed excellent DOX loading capacity and acidic $\mathrm{pH}$ stimulated controlled release of the drug. ${ }^{34}$ Fan et al. reported the synthesis of a graphene-CNT$\mathrm{Fe}_{3} \mathrm{O}_{4}$ nanoparticle hybrid $\left(\mathrm{GN}-\mathrm{CNT}-\mathrm{Fe}_{3} \mathrm{O}_{4}\right)$ and showed its pH-dependent drug release properties. ${ }^{35} 5$-FU loaded $\mathrm{GN}-\mathrm{CNT}-\mathrm{Fe}_{3} \mathrm{O}_{4}$ nanohybrids showed high toxicity against HepG2 cells in vitro. Another targeted $\mathrm{pH}$ responsive drug delivery system was reported by Song et al. comprising of HA linked GO nanosheets that showed an acidic $\mathrm{pH}$ responsive DOX release property, which caused $17 \%$ higher tumor inhibition in $\mathrm{H} 22$ hepatic carcinoma cell tumors in mice as compared to only GO loaded DOX groups. ${ }^{36}$ In addition to these studies, there are several other reports on the development of $\mathrm{pH}$ responsive graphene-based drug delivery systems that further highlight the potential and importance of graphene-based nanomaterials in drug delivery. ${ }^{37-39}$

Wen et al. exploited the high levels of glutathione (GSH) in cancer cells and tumors as a stimulus to induce drug release and developed a NGO-based drug delivery system consisting of a redox-responsive PEG shell attached to NGO sheets via disulfide linkages. ${ }^{40}$ In presence of GSH in the environment, the PEG shells got detached from the system due to disulfide bond breakage and caused 1.55 times faster drug release than in the absence of GSH (Fig. 2(iii)). Another study reported GSH-triggered anti-cancer drug release from PEGylated graphene oxide (PEG-GO) nanocarriers, both in in vitro and in vivo. ${ }^{41}$

\subsubsection{External stimuli responsive systems}

A NIR responsive photothermally triggered cytosolic drug delivery system was developed by Kim et al. using PEG and BPEI dual polymer functionalized rGO nanosheets (PEG-BPEI-rGO). ${ }^{42}$ The PEG-BPEI-rGO nanocomposites showed high drug loading efficiency with absorbance in the NIR region. Drug release was mediated by both GSH and photothermal effect of NIR light irradiation. It was observed that after cellular uptake, the nanocomposites escaped the endosomes via photothermally induced endosome disruption and proton sponge effect and subsequently released the drug into the cytosol in response to high GSH concentrations inside cancer cells (Fig. 2(iv)). This photothermal effect on the drug release was found to cause more cytotoxicity against cancer cells in comparison to those not irradiated with NIR light.

Graphene-based nanocomposites containing magnetite or iron oxide nanoparticles can be effectively used as a drug delivery system responsive to an external magnetic field. This was shown by Zhou et al. who synthesized graphene $/ \mathrm{Fe}_{3} \mathrm{O}_{4}$ composites through a simple one-pot solvothermal process. ${ }^{43}$ These graphene $/ \mathrm{Fe}_{3} \mathrm{O}_{4}$ composites showed good water dispersibility alongwith excellent magnetic properties ideal for a magnetically guided drug delivery system. Doxorubicin was loaded onto these composites with a high loading efficiency of about $65 \%$. The release of drugs from these composites could be controlled and guided by an external magnetic field. 
In another study, $\mathrm{Li}$ et al. reported the synthesis of $\mathrm{Fe}_{3} \mathrm{O}_{4}$ nanoflowers encapsulated in graphene nanosheets $\left(\mathrm{Fe}_{3} \mathrm{O}_{4} / \mathrm{GNS}\right)$ and demonstrated the feasibility of these nanocomposites to be used as drug delivery systems under the influence of an external magnetic field by loading Rhodamine B onto them with a high loading efficiency. ${ }^{44}$

Apart from magnetic field, electric field can also be used as a stimulus to control drug delivery. Liu et al. reported the development of an electrically modulated drug delivery system comprising of rGO sheets embedded in a matrix of PVA, forming a hydrogel. ${ }^{45}$ Lidocaine hydrochloride was loaded into this hydrogel as a model drug and its release properties were studied under the influence of an external electric field. In the absence of an electrical stimulus, drug release was not observed. Drug release occurred only in the presence of electric field, thereby demonstrating the electrical stimulus responsive properties of this hydrogel.

\section{Graphene-Based Nanomaterials for Nucleic Acid Delivery}

The most sought after vectors for carrying out efficient gene delivery to cells, both in vitro and in vivo have been viral vectors because of their very high transfection efficiency. ${ }^{46}$ However, concerns related to the safety and biocompatibility of these viral vectors such as chances of random genomic integration, activation of oncogenes, stimulation of unwanted immune response in the host and re-activation of the inactivated viruses have limited the large scale application and clinical translation of these vectors for gene therapy. ${ }^{46}$ So, for the development of safer vectors, researchers have moved towards non-viral approaches of gene delivery. ${ }^{47}$ Different types of materials such as cationic polymers, lipids, polysaccharides and dendrimers have been explored for the development of non-viral vectors, which demonstrated their abilities to carry and deliver genes into cells and tissues, however could not reach the high level of transfection efficiencies as shown by viral vectors. ${ }^{47}$ In addition to this, cytotoxicity and biocompatibility issues were also observed with cationic lipids and polymers due to their high density of surface positive charges that are required for efficient nucleic acid complexation. Thus, the search for an efficient and safe vector which can provide high level of transfection efficiency without inducing any toxicity or undesirable side-effects in the host is still on.

The structural, optical and biocompatible properties of graphene and its derivatives such as GO and rGO have raised a lot of interest in this material in the recent years, that have led to studies related to the synthesis and development of non-viral vectors using graphene-based materials as a novel carrier system for gene delivery. ${ }^{48}$ Small size, high surface area to mass ratio, easy surface functionalization, ability to load and condense nucleic acids even in the absence of cationic groups through $\pi-\pi$ interactions, protection of nucleic acids from endonuclease digestion, high cellular uptake and low toxicity has made graphene and its derivatives a suitable candidate for gene delivery. ${ }^{5}$ The ease of functionalization of these materials with cationic polymers and targeting moieties to improve their biocompatibility, nucleic acid 
loading efficiency and overall therapeutic efficacy is due to the presence of numerous oxygen containing functional groups on the surface of these materials that allow for easy conjugation and derivatization. ${ }^{5,8}$ Covalent and non-covalent functionalization of $\mathrm{GO}$ and $\mathrm{rGO}$ nanosheets with cationic polymers like polyethylenimine (PEI), polysaccharides like chitosan and dendrimers such as polyamidoamine (PAMAM) have been carried out to develop nanoconstructs with improved nucleic acid binding efficiency and physiological stability for achieving better transfection and therapeutic outcomes., ${ }^{8,48}$ The cationic molecules provide a net positive charge on the surface of the nanoconstructs that help in improving the nucleic acid condensation efficiency through electrostatic interactions. Incorporation of PEG alongwith these groups have also shown to improve the aqueous stability and biocompatibility of these nanoconstructs. In addition to this, these grafting strategies of cationic polymers and dendrimers onto the surface of GO and rGO also helped to minimize their cytotoxicity by decreasing the concentration of the positive charges in the nanoconstructs without affecting the efficient nucleic acid binding capacities.

The first attempt towards successful delivery of a nucleic acid using graphenebased nanomaterial as a vector was made by Lu et al. ${ }^{49}$ The group used NGO sheets to deliver a molecular beacon (MB) to HeLa cells in culture for intracellular sensing of the expression level of survivin gene at the mRNA level (Fig. 3(i)). A molecular beacon is basically a single stranded DNA folded into a hairpin structure having a self-complementary stem alongwith a single stranded loop that is complementary to a specific target. In the closed conformation, the stem brings together a fluorophore and a quencher attached to them in close proximity resulting in fluorescence quenching. However, in the presence of the target sequence, the MB undergoes a conformational change and opens up to hybridize with the target, thereby taking the fluorophore-quencher pair away from each other, which ultimately leads to restoration of the fluorescence. This fluorescence off/on mechanism is used as a probe to detect the presence of a target sequence. In this study, the authors observed that the NGO sheets not only showed efficient loading of the MB on its surface, but also protected it from endonuclease digestion. In addition to this, the MB retained its target sequence binding ability even after loading onto NGO which was evidenced by the appearance of fluorescence inside the HeLa cells, thereby also demonstrating successful intracellular delivery of the nucleic acid payload. Simultaneously in the same year, Wang et al. also demonstrated real-time biosensing of ATP in JB6 cells using an aptamer/GO nanocomplex. ${ }^{50}$ Apart from mRNA and biomolecule sensing, PEI grafted graphene nanoribbons (PEI-GNR) loaded with MB were also used as molecular probes by Dong et al. for sensing of miR-21 in cells. ${ }^{51}$ The PEI-GNR showed more efficient delivery of MB as compared to PEI-MWCNTs and PEI alone. In a follow up study by the same group, the authors developed a multifunctional poly (L-lactide)-Polyethylene glycol-grafted graphene quantum dot-based platform for intracellular sensing of miR-21 combined with fluorescent tracking of the internalization of the complexes using GQDs. ${ }^{52}$ 


\section{(i) Molecular Probing}

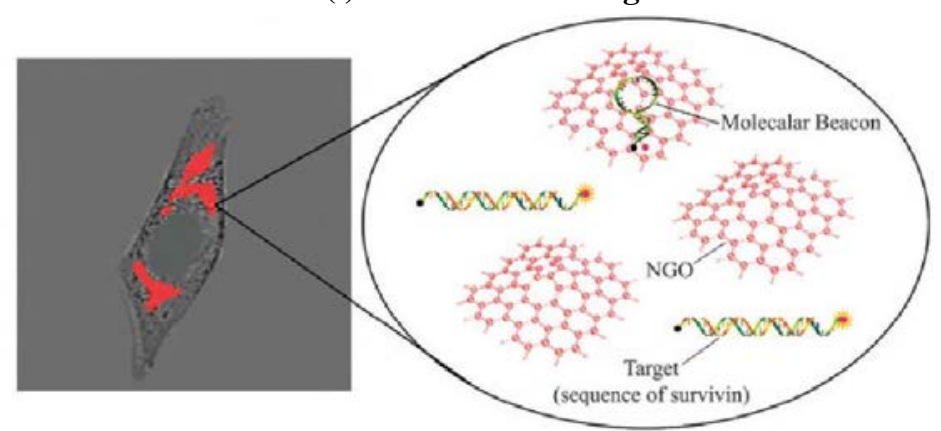

(ii) Gene Delivery

a)
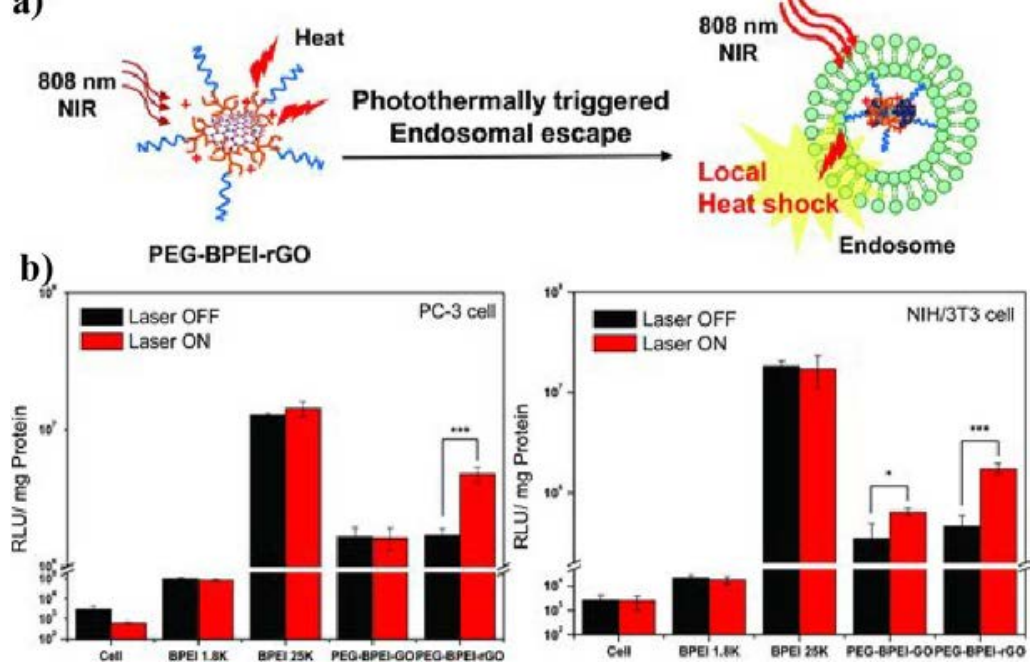

Fig. 3. Graphene-based nanomaterials for nucleic acid delivery. (i) Molecular probing. Schematic representation of NGO delivery of MB into HeLa cells to detect survivin mRNA. Reproduced with permission from Ref. 49. Copyright 2010 Royal Society of Chemistry. (ii) Gene delivery. (a) Schematic illustration of photothermal transfection mechanism. (b) Transfection of the BPEI (1.8 and $25 \mathrm{~K})$, PEG-BPEI-GO and PEG-BPEI-rGO with/without NIR irradiation in PC-3 and NIH/3T3 cell lines. Reproduced with permission from Ref. 60. Copyright 2013 Wiley-VCH Verlag GmbH \& Co. KGaA, Weinheim (iii) mRNA delivery. Efficient reprogramming from fibroblasts into iNs by GO-PEI-mRNA complexes; (a) Representative images and (b) percentages of Tuj1-positive iNs induced by lentiviral ABM, GO-PEI-ABM, and Lipofectamine ABM; (c) qRT-PCR analysis of the neuronal genes Tuj1, Map2, NeuroD1, and Syp in Lentiviral ABM and GO-PEI-ABM transduced fibroblasts; Three independent experiments were performed in triplicate. Data are presented as means \pm standard errors of the mean (SEM); Student's $t$-test; $* p<0.05, * * p<0.01$. (d) Immunofluorescence of Map2, NeuN, PSD97, and vGlut1 in GO-PEI-ABMinduced neurons; Scale bars, $100 \mu \mathrm{m}$. (e) Flow cytometry analysis of the induction of Tau-eGFP + cells by GO-PEI-ABM; proportions of Tau-eGFP+ cells among treated cells are indicated as percentages. (f) GOPEI-ABM complexes induced iNs expressed Syp, vGlut1, and Glutamate; Scale bars, $100 \mu \mathrm{m}$. (g) Number of Syp, vGlut1, and Glutamate positive iNs induced by Go-PEI-ABM complexes. (h) Confirmation of transgene integration using genomic-DNA PCR; Lane 1, Lipofectamine-ABM transfected fibroblasts; Lane 2, lentiviral-ABM infected fibroblasts; Lane 3, GO-PEI-ABM transfected fibroblasts. Reproduced with permission from Ref. 61. Copyright 2016 Wiley-VCH Verlag GmbH \& Co. KGaA, Weinheim. 


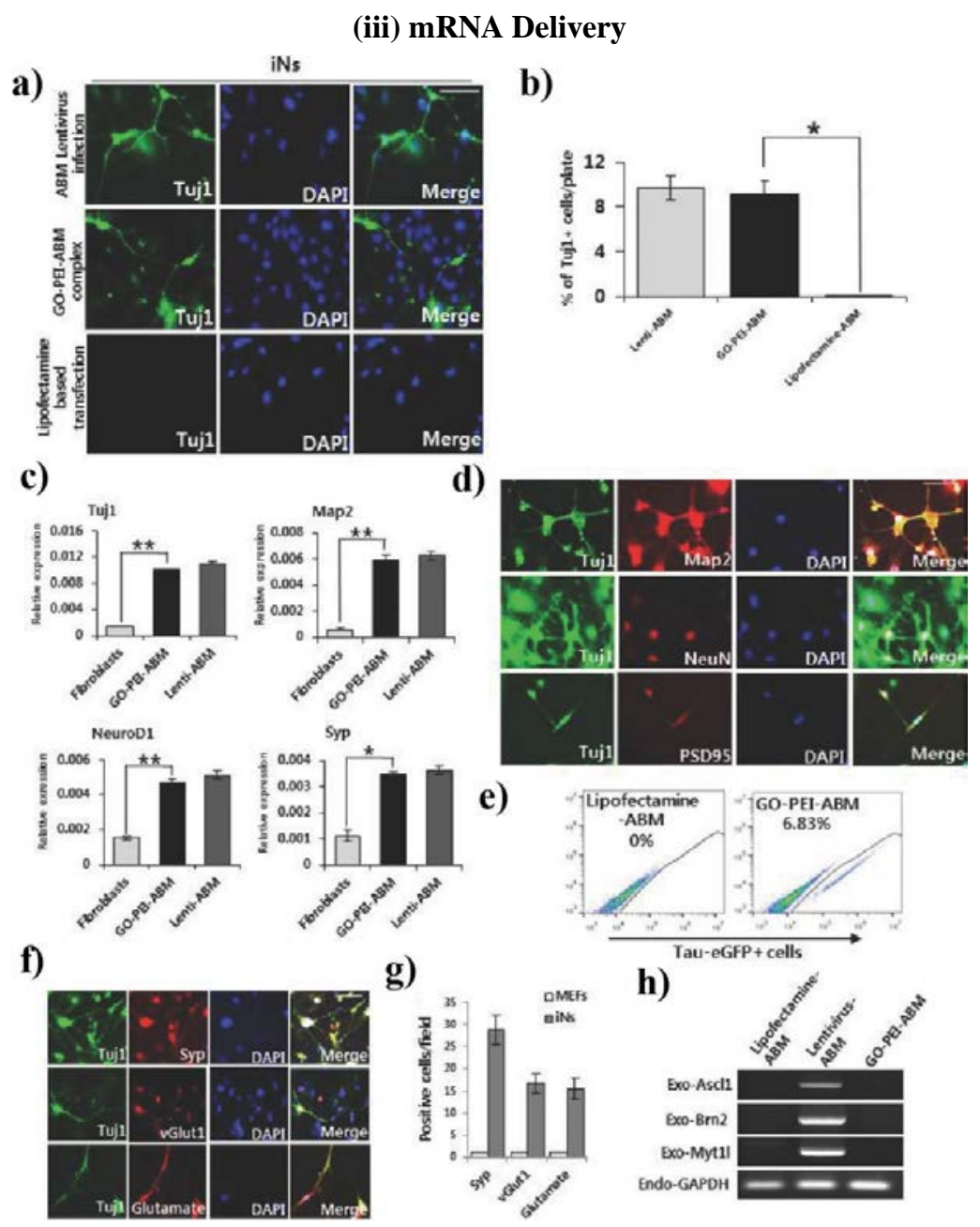

Fig. 3. (Continued)

In addition to molecular sensing, graphene-based nanovectors have been extensively used for the delivery of foreign genes encoded in plasmid DNAs for expression in cells. However, most of these works have focussed mainly on the delivery of plasmid DNA encoding a fluorescent protein like EGFP to demonstrate the loading and transfection efficiencies of different surface functionalized graphene-based nanovectors, without any emphasis on therapeutic application. For example, Chen et al. used GO sheets covalently functionalized with BPEI as a gene delivery tool for delivering plasmid DNA encoding EGFP to HeLa cells. ${ }^{53}$ Similarly, in another study, Kim et al. used PEI functionalized GO sheets for delivering luciferase gene into cells for bioimaging applications. ${ }^{54}$ Several other studies utilized this PEI-GO 
nanoconstruct as a vector and demonstrated highly efficient delivery of EGFP plasmid DNA into cells in vitro, either in a non-targeted or targeted manner. ${ }^{55-58}$ Recently, the concept of photothermally enhanced gene delivery was introduced for the first time by Feng et al. followed by a subsequent study by Kim et al., who independently showed that the transfection efficiency of graphene-based nanovectors could be improved significantly by exploiting the NIR responsive photothermal properties of GO and rGO at the time of transfection. ${ }^{59,60}$ Feng and co-workers developed a PEG-PEI dual polymer functionalized nGO-based composite (NGOPEG-PEI) and demonstrated highly efficient and greater EGFP transfection efficiency as compared to GO-PEI and PEI alone, in presence of low power NIR irradiation due to the localized photothermal heating that enhanced the cellular uptake of the nanocarriers as a result of increased cell membrane permeability, without inducing any cytotoxicity. ${ }^{59}$ They further utilized this nanocarrier for siRNA delivery into cells and demonstrated enhanced downregulation of the target gene (Plk1) in presence of NIR laser mediated transfection. In a subsequent work, Kim et al. reported the application of PEG-PEI-rGO nanocomposites for photothermally enhanced gene delivery and further demonstrated that the enhancement in transfection efficiency upon NIR irradiation was caused due to accelerated endosomal escape of polyplexes augmented by locally induced heat (Fig. 3(ii)). ${ }^{60} \mathrm{Li}$ et al. reported a new method of gene delivery using patterned nGO matrixes as cell culture substrates onto which PEI/pDNA complexes were adsorbed. These substrates showed gradual release of the PEI/pDNA complexes which resulted in transfection of the cells being cultured on those substrates. ${ }^{61}$ In a remarkable study conducted by Khademhosseini and group, PEI functionalized GO nanosheets (fGO) incorporated in methacrylated gelatin (GelMA) hydrogel was used as a carrier ( $\mathrm{fGO}_{\mathrm{VEGF}} / \mathrm{GelMA}$ ) for vascular endothelial growth factor-165 (VEGF) gene delivery for myocardial therapy. ${ }^{62}$ In a rat model of myocardial infarction, the $\mathrm{fGO}_{\mathrm{VEGF}} /$ GelMA hydrogels were injected intramyocardially in the peri-infarcted regions, which showed a significant increase in the microcapillary density in the infarcted region alongwith a reduction in scar area and improvement in cardiac function as compared to controls, without invoking any inflammatory and toxic responses in the host. This study demonstrated the immense potential of graphene-based materials to serve as nonviral vectors in gene therapy. In another equally interesting study published recently by Choi et al., GO-PEI nanocomposites were exploited for the first time as mRNA delivery vectors for the generation of "footprint-free" induced pluripotent stem cells (iPSCs). ${ }^{63}$ Footprint-free iPSCs are very important from a clinical point of view because their creation do not involve genomic integration of the reprogramming factor genes, thereby minimizing the risk of insertional mutagenesis in humans. GOPEI nanocomplexes showed highly efficient loading of mRNA of reprogramming transcription factors (Oct3/4, So2, Klf4, cMyc) and also provided protection to the mRNAs from RNAse degradation. In addition to this, the GO-PEI/RNA complexes could transfect adult adipose tissue-derived fibroblasts in suspension cultures with a high efficiency and successfully generated both rat and human iPSCs from them. The 
generated iPSCs showed all markers of pluripotency like expression of pluripotency genes, epigenetic reprogramming, and differentiation into the three germ layers. This study laid the framework for the application of graphene-based materials as non-viral mRNA delivery vectors for generation of iPSCs and for potential applications in direct cell-reprogramming-based therapies for the treatment of a wide variety of diseases. Two very recent works demonstrated the application of GO-PEI complexes as mRNA and gene delivery vectors for direct reprogramming of human somatic cells into cells of neural and mesodermal lineage. ${ }^{64,65}$ Baek et al. demonstrated the reprogramming of fibroblasts into integration-free induced neurons (iNs) through the transfer of mRNA of neural lineage specific reprogramming factors using GO-PEI complexes, that alleviated Parkinson's disease symptoms in a mouse model (Fig. 3 (iii)). ${ }^{64}$ In another study, Chiang et al. demonstrated the direct reprogramming of human peripheral blood mononuclear cells into cells of the mesodermal lineage using

Table 2. Summary of graphene-based nanomaterials for nucleic acid delivery.

\begin{tabular}{|c|c|c|c|}
\hline Nanocomposite & Nucleic acid cargo & Target & Reference \\
\hline NGO & $\begin{array}{l}\text { Molecular beacon for surviving gene } \\
\text { sensing }\end{array}$ & HeLa cells & 49 \\
\hline GO & Aptamer for ATP sensing & JB6 cells & 50 \\
\hline PEI-GNR & Molecular beacon for miR-21 sensing & HeLa cells & 51 \\
\hline PLA-PEG-GQD & $\begin{array}{l}\text { Molecular beacon for miR-21 sensing, } \\
\text { miR-21 and surviving targeting } \\
\text { antisense ODNs }\end{array}$ & HeLa cells & 52 \\
\hline BPEI-GO & pEGFP & HeLa cells & 53 \\
\hline PEI-GO & Luciferase gene & HeLa \& PC-3 cells & 54 \\
\hline PEI-GO & pEGFP & HeLa \& 293 T cells & 55 \\
\hline PEI-GO & pEGFP & $\begin{array}{l}\text { H293T, U20 cells, } \\
\text { zebrafish embryos }\end{array}$ & 56 \\
\hline PEI-GO & pEGFP & HeLa cells & 57 \\
\hline PEI-GO & pEGFP & HeLa cells & 58 \\
\hline NGO-PEG-PEI & pEGFP, Plk1 siRNA & HeLa cells & 59 \\
\hline PEG-PEI-rGO & Luciferase gene & NIH3T3 and PC-3 & 60 \\
\hline nGO matrix & pGFP, luciferase & $\begin{array}{l}\text { HEK293T, HeLa \& } \\
\text { hMSC }\end{array}$ & 61 \\
\hline $\begin{array}{l}\text { PEI-GO/GelMA } \\
\text { hydrogel }\end{array}$ & VEGF-165 gene & $\begin{array}{l}\text { Mouse model of myo- } \\
\text { cardial infarction }\end{array}$ & 62 \\
\hline PEI-GO & $\begin{array}{l}\text { mRNA of Oct } 3 / 4, \text { So } 2, \text { Klf } 4, \text { cMyc } \\
\text { transcription factors }\end{array}$ & $\begin{array}{l}\text { Adipose tissue derived } \\
\text { fibroblasts }\end{array}$ & 63 \\
\hline GO-PEI & $\begin{array}{l}\text { mRNA of neural lineage specific } \\
\text { transcription factors }\end{array}$ & Fibroblasts & 64 \\
\hline $\mathrm{GO}-\mathrm{Fe}_{3} \mathrm{O}_{4}$-PEI & $\begin{array}{l}\text { Episomal plasmids encoding pluripotent } \\
\text { related factors }\end{array}$ & PBMC & 65 \\
\hline GO-CS & Luciferase gene & HeLa cells & 66 \\
\hline GO-PAMAM & Luciferase gene & $\begin{array}{l}\text { HeLa \& MG63 } \\
\text { fibroblasts }\end{array}$ & 67 \\
\hline GO-PEI & pGFP \& anti-pGFP siRNA & HEK293 \& HeLa cells & 68 \\
\hline GO-PEI & anti-CXCR4 siRNA & MDA-MB-231 cells & 69 \\
\hline $\begin{array}{l}\text { rGO-PL-PEG- } \\
\text { Octarginine }\end{array}$ & Cell death siRNA & MCF-7 cells & 70 \\
\hline
\end{tabular}


GO-Fe $\mathrm{O}_{4}$-PEI complexes carrying three episomal plasmids encoding pluripotent related factors via a process of combined magnetic stirring and photothermal stimulation to enhance the transfection efficiencies of the complexes in suspension culture. ${ }^{65}$ The transfected PBMCs were reprogrammed into iPSCs, which rapidly transdifferentiated into mesodermal lineages without full reprogramming.

Chitosan functionalized GO nanovector was also explored as a gene delivery vector by Bao et al. for delivering luciferase gene into HeLa cells. ${ }^{6}$ However, the transfection efficiency of such vectors was not found to be as high as PEI. Cationic dendrimers like PAMAM have also been explored for gene delivery. Liu et al. developed a PAMAM and oleic acid functionalized GO nanocomposite and demonstrated transfection of HeLa and MG63 fibroblast cells with luciferase gene at a high transfection efficiency. ${ }^{67}$ These composites also showed good physiological stability.

Graphene-based nanomaterials have also been explored for delivering siRNAs to cells in vitro for downregulating the expression of specific genes that are found to be overexpressed in pathological conditions such as cancer. Tripathi et al. used GO-PEI nanocomplex for delivering GFP expressing pDNA to cells in culture and using the same vector delivered a anti-GFP siRNA $3 \mathrm{~h}$ later. ${ }^{68}$ The authors reported $70 \%$ knockdown in the fluorescence levels. Using a similar GO-PEI nanocomplex, Huang et al. recently demonstrated suppression in cancer cell migration through efficient knockdown of CXCR4 gene using anti-CXCR4 siRNA, which is strongly upregulated in cancer metastasis. ${ }^{69}$ In another recent study, Imani et al. reported the synthesis of a rGO-based nanocarrier functionalized with phospholipid-PEG and conjugated with a cell penetrating peptide octarginine. ${ }^{70}$ The nanocarrier was used to selectively deliver a cell death siRNA to MCF-7 cells in vitro. The nanocarriers showed good nucleic acid binding, endonuclease protection, high transfection efficiency, superior buffering capacity in the acidic $\mathrm{pH}$ range of 4-6 and induced $51 \%$ cell death in treated cells as compared to non-treated ones. Table 2 summarizes the different graphene-based nucleic acid delivery systems developed so far.

\section{Graphene-Based Nanomaterials for Phototherapy}

Graphene and graphene-based materials such as GO and rGO have emerged as excellent nanomaterials for carrying out photothermal and photodynamic therapies of cancer because of their surface chemistry and intrinsic optical properties. Photothermal therapy is a widely exploited non-invasive cancer therapy procedure that involves the absorption of light of a particular wavelength especially NIR by nanomaterials or dyes or a combination of both, which are capable of converting this light energy into heat for photothermal killing or ablation of cancer cells and tumors, both in in vitro and in vivo. ${ }^{71}$ Large surface area, presence of $s p^{2}$ clusters on the crystal lattice, strong absorption in the NIR region and small size have made both GO and 
rGO to act as excellent photothermal agents in response to NIR irradiation and generate heat through plasmonic photothermal conversion. ${ }^{72}$ The NIR absorbance of rGO has been found to be six times more as compared to GO because of the highly intact aromatic structure of rGO created during reduction process. On the other hand, photodynamic therapy that involves the use of light responsive photosensitizers as therapeutic agents for generation of highly cytotoxic reactive oxygen species (ROS) upon irradiation with light, have also attracted a great deal of attention in cancer therapy. GO and rGO have been used as scaffolds or carriers for loading and delivering these photosensitizers into cancer cells and tissues for photodynamic cancer therapy. ${ }^{73}$ The presence of oxygen containing functional groups on both surfaces of GO nanosheets make them ideal platforms for highly efficient loading of hydrophobic as well as hydrophilic photosensitizer molecules through several non-covalent interactions such as $\pi-\pi$ interactions, hydrophobic interactions, hydrogen bonding and electrostatic interactions. The following section describes the progress that has been made in the development of different kinds of graphene-based nanomaterials as efficient photoagents for successful photothermal and photodynamic therapy of cancers. Table 3 summarizes the different phototherapeutic applications of graphene-based nanomaterials.

\subsection{Photothermal therapy}

The Liu group was the first to report the photothermal properties of PEGylated nano-GO in a tumor model of mice. ${ }^{74}$ The group demonstrated that nGO-PEG exhibited enhanced absorption in the NIR region and caused complete photothermal ablation of tumors in mice, intravenously injected with the nanocomposites in response to NIR irradiation. Robinson et al. reported the use of rGO as a photothermal agent for the first time and showed that non-covalently PEGylated nanosized rGO sheets were more efficient in the photothermal conversion as compared to covalently PEGylated GO sheets. ${ }^{75}$ The group demonstrated targeted photothermal ablation of U87MG cancer cells in vitro by using RGD peptide tagged PEGylated nano-rGO in response to NIR irradiation. The nanocomposites showed negligible toxicity in the absence of NIR irradiation, thereby establishing itself as novel biocompatible photothermal agents for cancer therapy. The Liu group carried out a detailed study to understand the effects of size and surface chemistry of graphene sheets on the photothermal properties and further validated the findings of Robinson et al. that noncovalently PEGylated nanosized reduced graphene oxide sheets were more efficient in the photothermal conversion as compared to covalently PEGylated GO sheets. ${ }^{76}$ In their study, Liu and co-workers demonstrated 100\% photothermal ablation of tumors in mice injected with nRGO-PEG and irradiated with a low-power NIR laser $\left(0.15 \mathrm{~W} / \mathrm{cm}^{2}\right)$, which was one order of magnitude lower than that normally used for in vivo photothermal ablation by other nanomaterials. Following a different approach, Akhavan et al. reported a novel strategy for the synthesis of reduced graphene oxide by using glucose as both, reducing and functionalizing agent. ${ }^{77}$ 
Table 3. Summary of graphene-based nanomaterials for phototherapies.

\begin{tabular}{|c|c|c|c|}
\hline Nanocomposite & $\begin{array}{l}\text { Therapeutic } \\
\text { modality }\end{array}$ & Target & Reference \\
\hline nGO-PEG & PTT & $\begin{array}{l}4 \mathrm{~T} 1 \text { tumor bearing Balb/c mice, } \mathrm{KB} \text {, and } \\
\text { U87MG tumor bearing nude mice }\end{array}$ & 74 \\
\hline nRGO-PEG-RGD & PTT & U87MG cells & 75 \\
\hline nRGO-PEG & PTT & $4 \mathrm{~T} 1$ tumor bearing mouse & 76 \\
\hline RGO & PTT & LNCaP cells & 77 \\
\hline nGO-ThS & PTT & Amyloid fibrils & 78 \\
\hline RGO & PTT & HT29 \& SW48 & 79 \\
\hline $\mathrm{Cu}_{2} \mathrm{O}$ NC-RGO & PTT & HK-2, MDA-MB-231, A549 cells & 80 \\
\hline AuNS@RGO & PTT & HUVECs & 81 \\
\hline \multicolumn{4}{|l|}{ AuNR@RGO } \\
\hline AuNR-PEG-GO & PTT & A431 cells and tumors in mouse & 82 \\
\hline GON & PTT & AGS cells & 83 \\
\hline NGO-HA & PTT & Skin cancer tumors in mouse & 84 \\
\hline $\mathrm{ZnFe}_{2} \mathrm{O}_{4}-\mathrm{rGO}$ & PTT & $\begin{array}{l}\text { Prostate cancer in vitro, glioblastoma } \\
\text { tumors in vivo }\end{array}$ & 85 \\
\hline MUC-1 aptamer-AuNP-GO & PTT & MCF-7 cells & 86 \\
\hline GO-Cypate & PTT & $\begin{array}{l}4 \mathrm{~T} 1 \text { cells in vitro \& } 4 \mathrm{~T} 1 \text { tumor bearing } \\
\text { mice }\end{array}$ & 87 \\
\hline ZnPc-NGO-mPEG & PDT & MCF-7 cells & 88 \\
\hline Ce6-GO-FA & PDT & MGC803 cells & 89 \\
\hline GO-HB & PDT & $\begin{array}{l}\text { SGC-7901, SMMC-7721, HeLa, and A549 } \\
\quad \text { cells }\end{array}$ & 90 \\
\hline GO-HA & PDT & HeLa cells & 91 \\
\hline $\mathrm{GO} / \mathrm{TiO}_{2}$ & PDT & HeLa cells & 92 \\
\hline $\mathrm{ZnO}-\mathrm{GO}-\mathrm{FA}$ & PDT & HeLa cells & 93 \\
\hline Ce6-GO-HA & PDT & HeLa cells & 94 \\
\hline MB-GO & PDT & & 95 \\
\hline
\end{tabular}

The glucose reduced GO showed excellent biocompatibility and improved NIR absorbance as compared to hydrazine reduced GO, single and multi-walled CNTs and demonstrated efficient photothermal killing of LNCaP prostate cancer cells in vitro at very low concentrations $(0.05 \mathrm{mg} / \mathrm{ml})$. Li et al. exploited the NIR responsive photothermal properties of nano-GO sheets to induce dissociation of amyloid fibrils (A $\beta$ fibrils) by heat for the treatment of Alzhiemer's disease. ${ }^{78}$ The benzothiazole dye Thioflavin $\mathrm{S}$ (ThS) which has an affinity to selectively bind to $\mathrm{A} \beta$ fibrils instead of $\mathrm{A} \beta$ monomers was covalently linked to the nGO sheets resulting in the formation of GO-ThS-A $\beta$, which upon irradiation with a low-power NIR laser showed dissociation of the $\mathrm{A} \beta$ fibrils. The dissociation of the fibrils was monitored by the fluorescence change of ThS. In another study, green tea-rGO nanosheets (GT-rGO) were developed and used for the photothermal destruction of HT29 and SW48 colon cancer cells in vitro. ${ }^{79} \mathrm{Hou}$ et al. reported selective photothermal killing of cancer cells in vitro under visible light irradiation using water dispersible $\mathrm{Cu}_{2} \mathrm{O}$ nanocrystal-rGO hybrids as photothermal agents. ${ }^{80}$ In order to enhance the photothermal properties of GO and rGO, several groups have reported the development of plasmonic 
nanoparticle incorporated graphene-based nanocomposites. Lim et al. synthesized rGO coated plasmonic gold nanoshells and nanorods and demonstrated enhanced photothermal killing of cells in vitro as compared to non-coated or graphene oxidecoated gold nanoparticles. ${ }^{81}$ The enhanced photothermal efficiency of the nanocomposites can be attributed to the presence of plasmonic nanoparticles that significantly enhanced the NIR absorption efficiency of the nanocomposites. Gold nanorod-attached PEGylated graphene-oxide (AuNR-PEG GO) nanocomposites were synthesized by Dembereldorj et al. and used for photothermal therapy both in in vitro and in vivo. ${ }^{82}$ The presence of AuNRs significantly enhanced the NIR absorption efficiency of the nanocomposites which resulted in $40 \%$ reduction in cell viability of A431 epidermoid carcinoma cells and also reduced tumor volumes in mice. Femtosecond laser beam mediated photothermal destruction of cancer cells was demonstrated by $\mathrm{Li}$ et al. using graphene oxide nanoparticles (GONs). ${ }^{83}$ The GONs generated microbubbles upon exposure to femtosecond laser beam that facilitated the killing of cancer cells. Jung et al. reported photothermal ablation of skin cancer cells using hyaluronic acid conjugated nano GO sheets. ${ }^{84}$ The NGO-HA conjugates were delivered transdermally to tumor tissues in the skin of mice, where they showed increased tumor uptake and resulted in complete destruction of the tumors in response to a NIR laser without any further recurrence of tumors (Fig. 4(i)). Magneto-PTT of cancers was demonstrated by Akhavan et al. using zinc ferrite spinel $\left(\mathrm{ZnFe}_{2} \mathrm{O}_{4}\right)$-rGO nanocomposites $\left(\mathrm{ZnFe}_{2} \mathrm{O}_{4}\right.$-rGO) for carrying out in vitro and in vivo photothermal destruction of prostate cancer cells and glioblastoma tumors, respectively, under the guidance of a magnetic field. ${ }^{85}$ An aptamergold nanoparticle-hybridized graphene oxide nanocomposite was developed by Yang et al. for targeted photothermal destruction of MCF-7 cells. ${ }^{86} \mathrm{MCF}-7$ cells showed increased cellular uptake of the nanocomposites via specific interaction between MUC-1-binding aptamer and MUC-1 that are present on the cell membrane of MCF-7 cells. In the presence of NIR irradiation, the nanocomposites exhibited excellent photothermal ablation activity at very low concentrations. Recently, Guo et al. reported the synthesis of a novel $\mathrm{pH}$-responsive graphene oxide-based photothermal agent for improved photothermal killing of cancer cells. ${ }^{87} \mathrm{~A}$ cyanine dye (Cypate) was grafted onto the surface of GO, which participated in enhancing the photothermal properties of the composite via FRET in a $\mathrm{pH}$-responsive manner. Under NIR irradiation, the Cypate molecules emitted near-infrared fluorescence (NIRF) that got transferred to the GO sheets via FRET and subsequently got converted into heat for improving photothermal therapy. A single dose intravenous injection of the GO-Cypate nanocomposites into a mouse bearing tumors followed by NIR irradiation resulted in significant photothermal destruction of the tumors.

\subsection{Photodynamic therapy}

PDT using GO was reported for the first time by Dong et al. ${ }^{88}$ They used methoxy PEG conjugated nano graphene oxide (NGO-mPEG) as a platform for loading of a 


\section{(i) Photothermal Therapy}

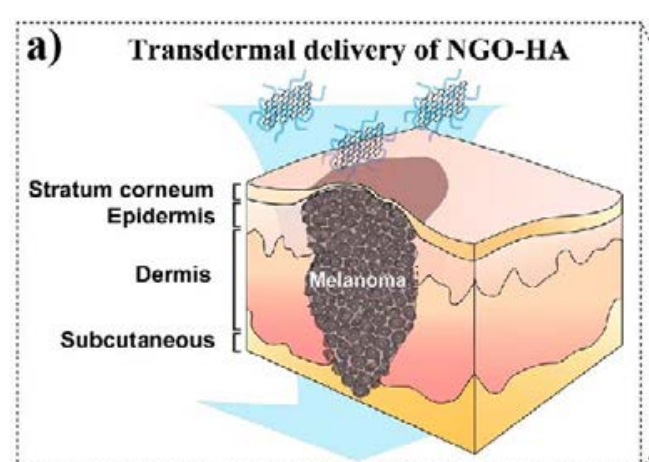

b)

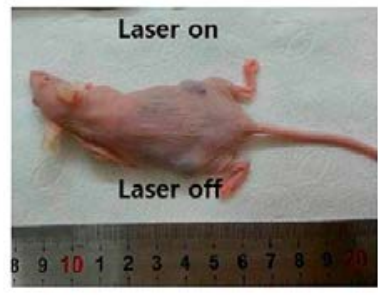

e)

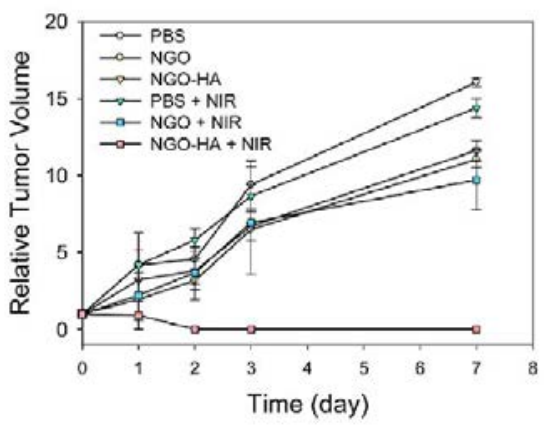

f) c)

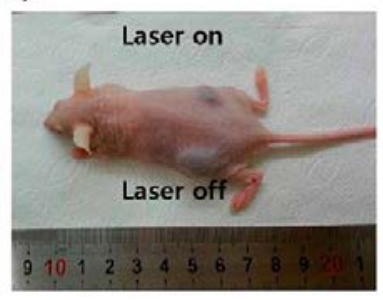

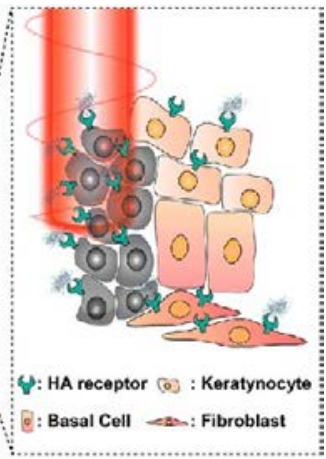

d)

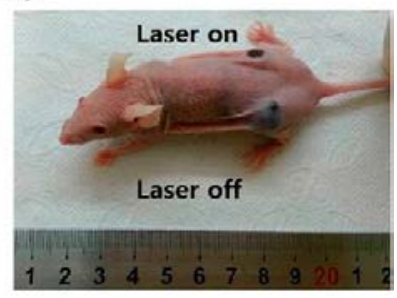

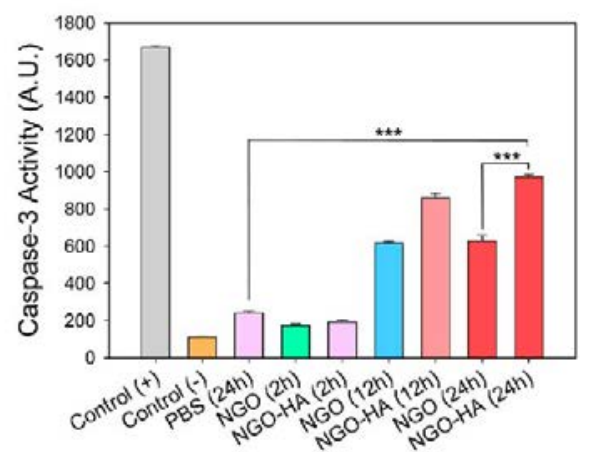

Fig. 4. Graphene-based nanomaterials for photo-therapy. (i) Photothermal therapy. (a) Schematic illustration for the transdermal delivery of nanographene oxide-hyaluronic acid (NGO-HA) conjugates into melanoma skin cancer cells and the following photothermal ablation therapy using a NIR laser. Photographs showing the effect of photothermal ablation therapy with NIR irradiation on the tumor growth in SKH-1 mice inoculated with B16F1 cells on both dorsal flanks after topical administration of (b) PBS, (c) NGO, and (d) NGO-hyaluronic acid (HA). (e) Relative tumor volume $\left(V / V_{0}\right)$ with increasing time for a week. (f) Caspase-3 activity in tumor tissues by ELISA with increasing time for a day for the analysis of heat induced apoptosis $(* * * P<0.0001)$. Reproduced with permission from Ref. 84 . Copyright 2014 American Chemical Society. (ii) Photodynamic therapy. MTT cell viability of HeLa cells treated with HA-GO/Ce6 nanohybrids $(\square)$ or free $\mathrm{Ce} 6(\bullet)$ for $6 \mathrm{~h}$, followed by the light irradiation of $1.8 \mathrm{~J} \mathrm{~cm}^{-2}$ (a), or without the light irradiation (b); LIVE/DEAD cell viability of HeLa cells treated with HA-GO/Ce6 nanohybrids or free Ce6 at a Ce6 concentration of $0.1 \mu \mathrm{g} \mathrm{mL}{ }^{-1}$ for $6 \mathrm{~h}$ of treatment (c). Reproduced with permission from Ref. 94. Copyright 2013 Royal Society of Chemistry. 
(ii) Photodynamic Therapy
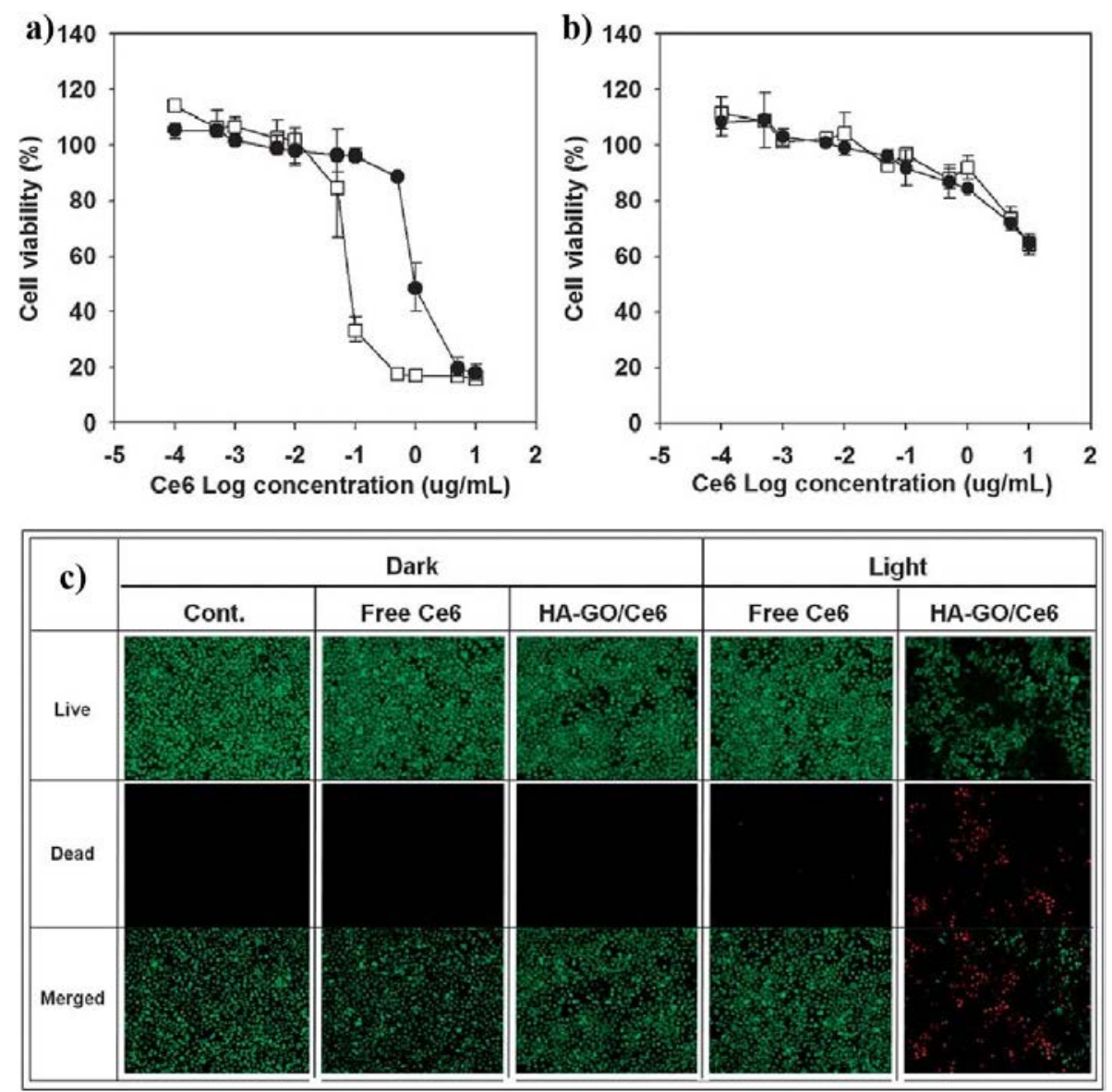

Fig. 4. (Continued)

photosensitizer zinc phthalocyanine $(\mathrm{ZnPc})$ onto the surface of NGO through $\pi-\pi$ and hydrophobic interactions. The ZnPc-NGO-mPEG nanocomposites demonstrated significant cytotoxicity in MCF-7 cell lines in response to Xe light irradiation. Huang et al. reported the development of a targeted photodynamic agent by conjugating folic acid with GO sheets and loading it with the photosensitizer Chlorin e6 (Ce6). ${ }^{89}$ The loading efficiency of Ce6 was found to be $80 \%$, with FA conjugation helping in increasing the accumulation of the nanocomposites in MGC803 cells. The nanocomposites exhibited excellent targeted photodynamic killing of MGC803 cells in response to laser irradiation. Hypocrellin A (HA) and Hypocrellin B (HB) are another class of organic photosensitizers that have the potential to be used in PDT, but have gained limited attention due to their stability issues in aqueous conditions. 
In order to improve their stability, Zhou et al. loaded HA and HB onto the surface of GO nanosheets (GO-HA and GO-HB) through hydrogen bonding and $\pi-\pi$ interactions and observed improved stability of $\mathrm{HA}$ and $\mathrm{HB}$ as compared to their free forms in aqueous solutions. ${ }^{90,91}$ Further, in vitro studies using these GO-HA and GO-HB nanocomposites demonstrated excellent photodynamic damage to cancer cells in response to light. In addition to organic photosensitizers, inorganic photosensitizers such as $\mathrm{TiO}_{2}$ and $\mathrm{ZnO}$ have also been explored for UV and visible light mediated PDT. Hu et al. developed a $\mathrm{GO} / \mathrm{TiO}_{2}$ hybrid and observed significantly increased production of intracellular ROS by the $\mathrm{GO} / \mathrm{TiO}_{2}$ hybrids when exposed to visible light. ${ }^{92}$ The authors explained that this enhanced photodynamic activity of $\mathrm{GO} / \mathrm{TiO}_{2}$ hybrids could be attributed to the excellent electrical conductivity of GO, which allowed the transfer of the photoelectrons generated by $\mathrm{TiO}_{2}$ in response to light to the surface of GO that ultimately reacted with oxygen to generate ROS. In another study by the same group, folic acid-conjugated graphene$\mathrm{ZnO}$ nanohybrid were synthesized for targeted PDT which showed efficient generation of ROS in HeLa cells in response to visible light irradiation, that ultimately resulted in apoptosis. ${ }^{93} \mathrm{Li}$ et al. reported the synthesis of hyaluronic acidconjugated GO nanocomposites loaded with Ce6 for targeted PDT of cancer cells in vitro and observed 10-fold increase in the PDT efficiency as compared to free Ce6 molecules (Fig. 4(ii)). ${ }^{94}$ Methylene blue (MB) loaded GO nanosheets have also been exploited as a PDT agent by Wojtoniszak et al., where the authors reported enhanced efficiency in singlet oxygen generation by MB-GO as compared to pristine MB. ${ }^{95}$

\section{Graphene-Based Nanomaterials for Combined Therapeutic Strategies}

\subsection{Combined drug delivery and phototherapies}

\subsubsection{Combined chemo-PTT}

The ability to load drugs efficiently onto the surface of graphene-based nanomaterials like GO and rGO due to the presence of a large surface area and also because of the presence of different oxygen containing functional groups, combined with their excellent absorbance in the NIR region have led to the development of multifunctional therapeutic platforms capable of providing synergistic chemo-PTT for better treatment of cancers. Zhang et al. developed a simple platform consisting of PEGylated NGO sheets loaded with the anti-cancer drug doxorubicin and demonstrated efficient killing of cancer cells both in vitro and in vivo. ${ }^{96}$ The therapeutic efficiency of the combined treatment strategy was found to be much higher as compared to chemotherapy and PTT alone. Folic acid conjugated, polyvinylpyrrolidone functionalized nanographene oxide sheets (FA-NGO-PVP) loaded with doxorubicin were synthesized by Qin et al., which showed highly efficient targeted delivery of drug and generated heat for effective chemo-photothermal 
destruction of tumors. ${ }^{97}$ Another targeted platform was developed by Yang et al. comprising of PEGylated NGO conjugated with EGFR antibody for targeting and loaded with the anti-cancer drug epirubicin for carrying out a synergistic tripletherapeutic treatment strategy involving growth signal blocking, chemotherapy and photothermal therapy. ${ }^{98}$ The NIR absorbance efficiency of GO and rGO has been found to be enhanced remarkably by incorporating plasmonic nanoparticles like AuNPs into the nanocomposite design. Based on this concept, Xu et al. synthesized a highly efficient chemo-photothermal therapeutic agent consisting of gold nanorods (AuNR) encapsulated in NGO shells and conjugated with hyaluronic acid for targeting. ${ }^{99}$ The NGO-HA-AuNR nanocomposites demonstrated 1.5 fold higher killing of Hu-7 cancer cells than chemotherapy alone and four fold higher photothermal ablation as compared to photothermal therapy alone. Wang et al. synthesized mesoporous silica coated graphene nanosheets conjugated with a targeting peptide (IP) and loaded with DOX as a chemo-photothermal agent for targeted therapy of glioma. ${ }^{100}$ The nanocomposites exhibited high drug loading efficiency along with sustained release profiles in response to both $\mathrm{pH}$ and NIR mediated heat. The therapeutic efficiency of the nanocomposites towards killing of glioma cells was found to be significantly enhanced due to the synergistic effect of chemo-photothermal therapy (Fig. 5(i)). A newer nanocomposite referred to as nanocookies was synthesized by Chen et al. that comprised of amorphous carbon coated mesoporous silica self-assembled on a nanosheet of reduced graphene oxide. ${ }^{101}$ These nanocookies were loaded with camptothecin and were used as novel chemo-photothermal agents for tumor treatment. A novel type of nanocapsule made up of lactoferrin shells stabilized by rGO was prepared by $\mathrm{Hu}$ et al. as a chemo-photothermal agent. ${ }^{102}$ Anti-cancer drugs could be efficiently loaded into these nanocapsules and could be used for both in vitro and in vivo chemo-photothermal treatment of cancer with high therapeutic efficiency. In addition to all these studies, several other graphene-based nanotherapeutic platforms such as CuS nanoparticle decorated GO, PEGylated graphene nanoribbons, $\mathrm{Fe}_{3} \mathrm{O}_{4}$ nanoparticle loaded $\mathrm{GO}$ nanocomposites have been developed for combined chemo-photothermal therapy of cancer. ${ }^{103-108}$

\subsubsection{Combined chemo-photodynamic therapy}

GO has also been used as a multimodal therapeutic platform for co-delivery of anticancer drugs and photosensitizers to cancer cells and tumors for synergistic chemophotodynamic therapy of cancer. Miao et al. reported the use of PEGylated GO nanosheets for co-delivery of doxorubicin and Ce6 to cancer cells both in vitro and in vivo. ${ }^{109}$ Both in vitro and in vivo studies showed enhanced therapeutic effect of the combined therapy in comparison to GO mediated chemotherapy and PDT alone. In a similar study, Zhou et al. showed a synergistic chemo-PDT of cancer by using GO sheets co-loaded with the anti-cancer drug 7-ethyl-10-hydroxycamptothecin (SN-38) and photosensitizer HA and observed significantly enhanced therapeutic effects of the combined therapy. ${ }^{110}$ 


\subsection{Combined gene delivery and phototherapies}

Graphene-based nanocomposites functionalized with cationic polymers have been developed and exploited as gene delivery vectors. Some very recent works have also exploited these positively charged graphene nanocomposites as multifunctional therapeutic platforms for combined gene-PTT with the ultimate objective of improving the therapeutic efficiency for better treatment of diseases like cancer. Cheng et al. reported the synthesis of PEGylated PEI-grafted graphene/Au composites (PPGA) as novel multifunctional gene delivery vectors and demonstrated efficient loading and delivery of Bcl-2 siRNA into HL-60 cells with subsequent
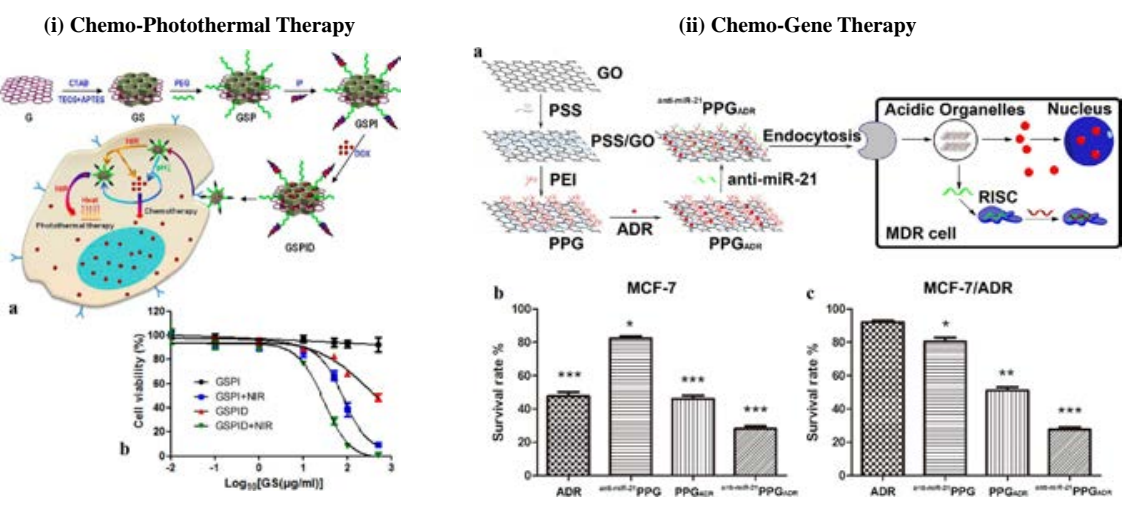

Fig. 5. Graphene-based nanomaterials for combined therapies. (i) Chemo-photothermal therapy. (a) Design of GSPID As a Multifunctional Drug Delivery System for Combined Chemo-Photothermal Targeted Therapy of Glioma (b) Cell viability profiles of glioma cells under different treatments. Reproduced with permission from Ref. 100. Copyright 2013 American Chemical Society. (ii) Chemogene therapy. (a) Schematic of the PPG fabrication and MDR reversion. Relative survival rate of MCF-7 cells (b) and MCF-7/ADR cells (c) after being treated with ADR, anti-miR-21PPG, PPGADR and anti-miR-21PPGADR for $24 \mathrm{~h}$. Untreated MCF-7 and MCF-7/ADR cells were served as control in each experiment. Reproduced with permission from Ref. 114. Copyright 2013. (iii) Chemo-gene-photothermal therapy. (a) Targeted gene-mediated thermochemotherapy for drug-resistant tumor. Apoptosis rate of MCF-7/ADR cells treated by (b) PBS (-), (c) Dox (-), (d) PPG-FA (+), (e) PPG-FA/ Dox (+), (f) PPG-FA/siRNA/Dox (-) and (g) PPG-FA/siRNA/Dox $(+)$ were tested by flow cytometry. They are measured by the average fluorescent intensity (FL) treated with Annexin V-FITC/PI. $(+)$; $808 \mathrm{~nm}$ irradiation $\left(2 \mathrm{~W} / \mathrm{cm}^{2}, 5 \mathrm{~min}\right)$; (-); without irradiation. Reproduced with permission from Ref. 115. Copyright 2017. (iv) Photothermal-photodynamic therapy. Photothermally enhanced delivery of PDT by graphene. (a)-(c) Confocal images of KB cells incubated with GO-PEG-Ce6 ( $5 \mu \mathrm{M}$ of $\mathrm{Ce} 6$ equivalent) at $37^{\circ} \mathrm{C}$ with (b) or without (a) $808 \mathrm{~nm}$ laser irradiation $\left(360 \mathrm{~J} / \mathrm{cm}^{2}\right)$ and cells incubated at $43^{\circ} \mathrm{C}$ in the dark (c). (d) and (e) Cell uptake of GO-PEG-Ce6 under the three conditions at $\mathrm{Ce} 6$ concentrations of $2.5 \mu \mathrm{M}$ (d) and $5 \mu \mathrm{M}$ (e) determined by the measured fluorescence intensities of cell lysate samples. (f) and (g) Cell viability data of KB cells incubated with GO-PEG-Ce6, free Ce6, or GO-PEG, respectively, at Ce6 concentrations of $2.5 \mu \mathrm{M}$ (f) and $5 \mu \mathrm{M}(\mathrm{g})$. Black, red, blue, and green bars represent samples without any light exposure, with both $808 \mathrm{~nm}\left(360 \mathrm{~J} / \mathrm{cm}^{2}\right)$ and $660 \mathrm{~nm}$ $\left(15 \mathrm{~J} / \mathrm{cm}^{2}\right)$ light irradiation, with only $660 \mathrm{~nm}$ light exposure, and with only $808 \mathrm{~nm}$ light exposure, respectively. Relative cell viabilities in all samples were normalized to the control saline-added samples without laser irradiation (100\% viability). Reproduced with permission from Ref. 116. Copyright 2011 American Chemical Society. 
(iii) Chemo-Gene-Photothermal Therapy

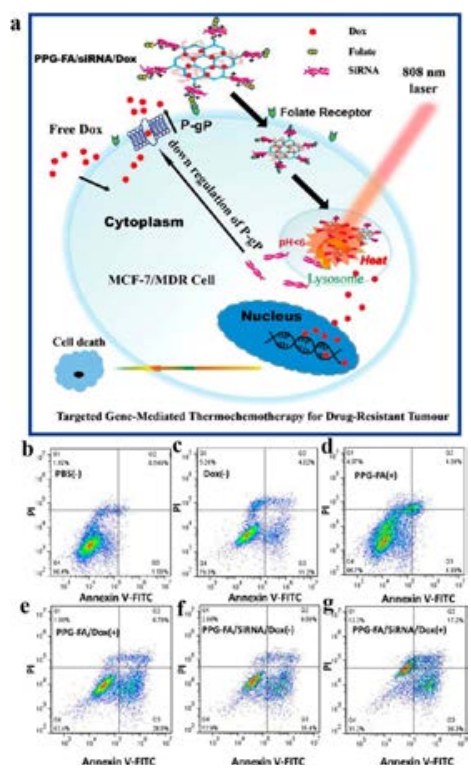

(iv) Photothermal-Photodynamic Therapy
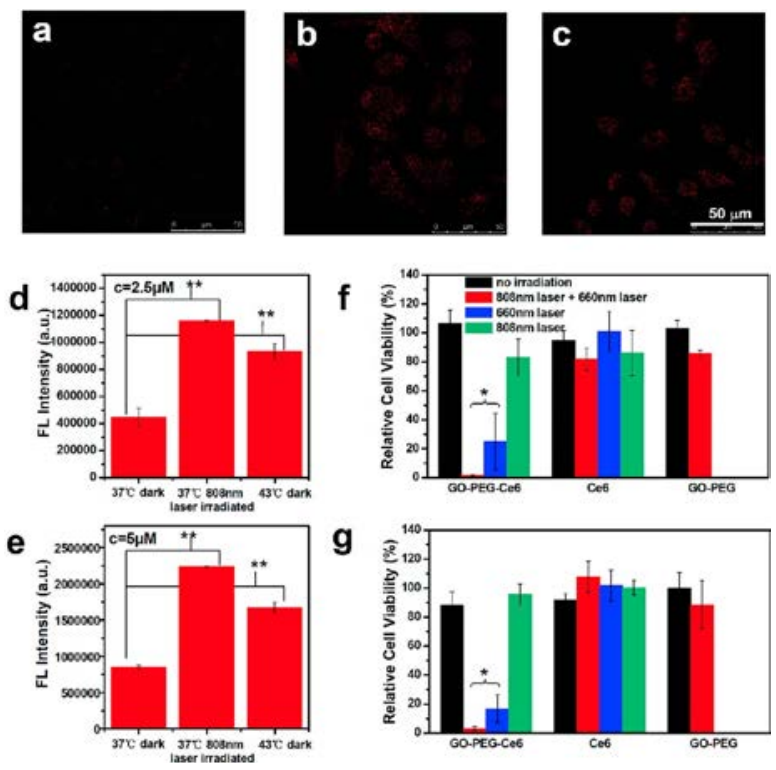

g

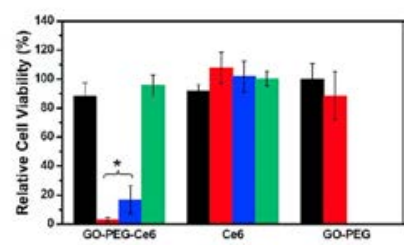

Fig. 5. (Continued)

downregulation of the Bcl-2 protein expression. ${ }^{111}$ In addition to this, the PPGA nanocomposites showed enhanced photothermal activity in response to NIR irradiation as compared to PPG nanocomposites lacking the AuNPs, thereby making it also an efficient photothermal agent. A PEG and PEI dual-polymer-functionalized GO nanocomposite (GO-PEG-PEI) was developed by Tao et al. for the delivery of immunostimulatory oligonucleotides like $\mathrm{CpG}$ both in in vitro and in vivo. ${ }^{112}$ It was observed that GO-PEG-PEI nanocomposites were successful in promoting the production of pro-inflammatory cytokines as a response to $\mathrm{CpGs}$. In addition to this, the researchers also observed that in the presence of NIR irradiation, the immunostimulation responses were much more enhanced possibly because of the photothermal properties of GO that generated local heat which resulted in accelerating the intracellular trafficking of the nanocomposites. The in vivo studies revealed efficient tumor reduction in the presence of NIR irradiation, which can be attributed to combined effects of both immunostimulation and photothermal ablation. In a very recent study published by Yin et al., PEGylated GO nanosheets have been used as a gene delivery vector for carrying out co-delivery of two siRNAs targeting the HDAC1 gene and the G12C mutant K-Ras gene in pancreatic cancer cells MIA PaCa-2. ${ }^{113}$ The PEGylated GO nanosheets successfully delivered the siRNAs to the cancer cells in vitro which ultimately resulted in induction of apoptosis and cell cycle arrest in the cells. In addition to this, in vivo studies demonstrated more than $80 \%$ inhibition 
in tumor growth under the influence of NIR irradiation due to a combined photothermal-gene silencing effect.

\subsection{Combined drug, gene and photo-therapies}

Zhi et al. developed a treatment strategy to overcome multi-drug resistance (MDR) in breast cancer tumors through co-delivery of an anti-cancer drug with a MDR reversing agent such as miRNA. ${ }^{114} \mathrm{PEI}$ and poly (sodium 4-styrenesulfonates) (PSS) dual-polymer-functionalized GO nanocomposites (PPG) were synthesized and sequentially loaded with adriamycin (ADR) and anti-miRNA-21 to form (anti-miR21)-PPGADR nanocomplexes. These nanocomposites successfully delivered the therapeutic agents into ADR resistant MCF-7 cells and showed enhanced cytotoxicity to the cancer cells as compared to free ADR. The enhanced therapeutic efficiency of these nanocomposites was due to the combined effects of both chemotherapy and gene silencing, thereby making the ADR resistant cells responsive to the drug (Fig. 5(ii)). In a very recent study, Zeng and co-workers developed a multifunctional PEI-PEG-GO delivery system conjugated with folic acid and co-loaded with a siRNA and Dox for carrying out targeted gene silencing combined with chemo-PTT of drug resistant tumors. ${ }^{115}$ In the absence of NIR irradiation, the nanocomposites showed gene silencing mediated enhanced chemotherapy of drug resistant cancer cells; while in the presence of NIR irradiation, the overall therapeutic efficiency was further enhanced due to the NIR responsive photothermal properties of GO (Fig. 5(iii)).

\subsection{Combined photothermal (PTT) and photodynamic (PDT) therapies}

Both visible and NIR responsive properties of GO and rGO have enabled them to be used as phototherapeutic agents for combined PTT and PDT. Tian et al. for the first time showed that the photodynamic properties of GO can be significantly enhanced by incorporating the NIR responsive photothermal properties of GO into a single multifunctional therapeutic platform. ${ }^{116}$ In this study, the authors showed that GOPEG-Ce6 complexes offered significant photodynamic destruction of cancer cells as compared to free Ce6 molecules. In addition to this, it was also observed that the NIR responsive photothermal effect of $\mathrm{GO}$ can be used to promote the delivery of Ce6 molecules, thereby further enhancing the PDT effect (Fig. 5(iv)). In another important study, Sahu et al. reported the development of a multifunctional phototherapeutic platform based on nano GO sheets non-covalently functionalized with a Pluronic block copolymer and loaded with the photosensitizer MB via electrostatic interactions. ${ }^{117}$ In vitro studies showed increased cellular uptake of the nanocomposites by cancer cells as compared to normal cells, and significant cell death in response to NIR irradiation. In vivo studies also depicted high tumor accumulation of the nanocomposites and complete tumor ablation through a combined PTT-PDT approach. Photothermal enhancement of the photodynamic efficiencies of 
graphene-based nanocomposites was also demonstrated by Chen et al. ${ }^{118}$ The group synthesized reduced graphene oxide- $\mathrm{ZnO}$ nanoparticles-hyaluronic acid (rGo-ZnOHA)-based multifunctional nanoplatform for a synergistic PTT-PDT of cancer. Visible light irradiation resulted in the generation of ROS by the $\mathrm{ZnO}$ nanoparticles, which helped in sensitizing the cancer cells for a subsequent NIR laser induced hyperthermia that resulted in more efficient killing of the cancer cells. Jiang et al. synthesized a water-soluble graphene-phthalocyanine (GR-TSCuPc) hybrid material by a one-step method involving simple mixing of graphene with tetrasodium salt copper phthalocyanine $(\mathrm{TSCuPc})$ through sonication. ${ }^{119}$ In the hybrid, graphene acted as the carrier of the photosensitizer and as a PTT agent, and TSCuPc served the role of a water-soluble PDT agent. In vitro studies showed that the GR-TSCuPc hybrid showed enhanced phototherapeutic effect as compared to free TSCuPc. Table 4 summarizes the applications of different graphene-based nanomaterials for combined therapeutic strategies.

Table 4. Summary of graphene-based nanomaterials for combined therapeutic strategies.

\begin{tabular}{|c|c|c|c|}
\hline Nanocomposite & Therapeutic modalities & Therapeutic cargo & Reference \\
\hline PEG-NGO & Drug delivery \& PTT & DOX & 96 \\
\hline FA-NGO-PVP & Drug delivery \& PTT & DOX & 97 \\
\hline PEG-NGO-EGFR & Drug delivery \& PTT & Epirubicin & 98 \\
\hline NGO-HA-AuNR & Drug delivery \& PTT & DOX & 99 \\
\hline $\mathrm{mSiO}_{2}$-GNS-IP & Drug delivery \& PTT & DOX & 100 \\
\hline $\begin{array}{l}\mathrm{RGO} / \text { Carbon } / \mathrm{mSiO}_{2} \\
\text { Nanocookies }\end{array}$ & Drug delivery \& PTT & $\mathrm{CPT}$ & 101 \\
\hline $\begin{array}{l}\text { Lactoferrin stabilized RGO } \\
\text { nanocapsule }\end{array}$ & Drug delivery \& PTT & DOX & 102 \\
\hline $\mathrm{PEG}-\mathrm{GO} / \mathrm{CuS}$ & Drug delivery \& PTT & DOX & 103 \\
\hline NrGO/PEG & Drug delivery \& PTT & Resveratrol & 104 \\
\hline $\begin{array}{l}\text { rNGO@mSiO }{ }_{2} @ \text { pNIPAM- } \\
\text { co-pAAm }\end{array}$ & Drug delivery \& PTT & DOX & 105 \\
\hline NGO-PEG-DA & Drug delivery \& PTT & DOX & 106 \\
\hline PL-PEG-GONR & Drug delivery \& PTT & DOX & 107 \\
\hline $\mathrm{GO}-\mathrm{Fe}_{3} \mathrm{O}_{4}$ & Drug delivery \& PTT & CPT \& MTX & 108 \\
\hline PEG-GO & Drug delivery \& PDT & DOX \& Ce6 & 109 \\
\hline GO & Drug delivery \& PDT & SN-38 \& HA & 110 \\
\hline $\begin{array}{l}\text { PEG-PEI-Graphene-Au } \\
\quad \text { (PPGA) }\end{array}$ & Gene delivery \& PTT & Bcl-2 siRNA & 111 \\
\hline GO-PEG-PEI & Gene delivery \& PTT & CpG ODNs & 112 \\
\hline PEG-GO & Gene delivery \& PTT & HDAC1 \& K-RAS siRNA & 113 \\
\hline PEI-PSS-GO & $\begin{array}{c}\text { Drug delivery, Gene } \\
\text { delivery \& PTT }\end{array}$ & ADR \& anti-miR-21 & 114 \\
\hline PEI-PEG-GO & $\begin{array}{c}\text { Drug delivery, Gene } \\
\text { delivery \& PTT }\end{array}$ & DOX \& siRNA & 115 \\
\hline PEG-GO & PTT \& PDT & Ce6 & 116 \\
\hline Pluronic F127-NGO & PTT \& PDT & Methylene blue & 117 \\
\hline rGO-ZnO-HA & PTT \& PDT & $\mathrm{ZnO} \mathrm{NPs}$ & 118 \\
\hline $\mathrm{GR}-\mathrm{TSCuPc}$ & PTT \& PDT & $\mathrm{TSCuPc}$ & 119 \\
\hline
\end{tabular}




\section{Graphene-Based Nanomaterials for Bioimaging}

In addition to being used as carriers for delivering therapeutic drugs and nucleic acids to cells and as NIR responsive agents for photothermal and photodynamic therapy, graphene-based nanomaterials have also been extensively exploited for bioimaging of cells and tissues. The intrinsic photoluminescence and NIR responsive properties of graphene and its derivatives alongwith its biocompatibility makes them suitable for bioimaging applications. Graphene-based bioimaging can be grouped into two major categories: - optical and non-optical imaging. ${ }^{120}$ Optical imaging includes fluorescence imaging, two-photon fluorescence imaging and Raman imaging, whereas non-optical imaging includes magnetic resonance imaging (MRI), radioactive imaging like PET and SPECT, photoacoustic imaging (PAI) and computed tomography $(\mathrm{CT})$. The following section summarizes the different imaging applications of graphene-based nanomaterials. In this section, studies related to only imaging using graphene and its derivatives have been reported. Studies related to imaging combined with different therapeutic approaches have been summarized in a later section that includes PAI and CT-based imaging using graphene. Table 5 summarizes the applications of graphene-based nanomaterials in bioimaging.

\subsection{Fluorescence imaging}

The intrinsic optical properties of graphene oxide make them suitable for fluorescence-based bioimaging of cells and tissues. The fluorescent properties of GO and

Table 5. Summary of graphene-based nanomaterials for bioimaging.

\begin{tabular}{|c|c|c|c|}
\hline Nanocomposite & Imaging modality & Target & Reference \\
\hline GO, nGO-PEG & Fluorescence & Raji B cells & 121 \\
\hline nGO-PEG-Cy7 & Fluorescence & Tumor xenografted mouse model & 74 \\
\hline GO-PEG-Fluorescein & Fluorescence & HeLa cells & 122 \\
\hline GO-IRDye800-VEGF & Fluorescence & Ischemic muscles in a murine model & 123 \\
\hline N-GQD & TPFI & Tissue phantom & 126 \\
\hline FA-GO-AgNP & Raman & A549 \& HeLa cells & 130 \\
\hline GO-AuNP & Raman & Ca Ski cells & 131 \\
\hline $\begin{array}{l}\text { Alkyne-PEG functionalized } \\
\text { graphitic silver nano- } \\
\text { particles (ACG NPs) }\end{array}$ & Raman & MCF-7 cells & 132 \\
\hline Gd-GNR & MRI & & 133 \\
\hline $\mathrm{AMD}-\mathrm{Fe}_{3} \mathrm{O}_{4}-\mathrm{GO}$ & MRI & HeLa cells & 134 \\
\hline $\begin{array}{l}{ }^{64} \mathrm{Cu}-\mathrm{NOTA}-\mathrm{nGO}-\mathrm{PEG}- \\
\mathrm{TRC} 105\end{array}$ & PET & 4T1 murine breast tumors & 136 \\
\hline $\begin{array}{l}{ }^{66} \text { Ga-NOTA-nGO-PEG- } \\
\text { TRC105 }\end{array}$ & PET & $4 \mathrm{~T} 1$ murine breast tumors & 137 \\
\hline${ }^{198,199} \mathrm{Au}-\mathrm{NGO}$ & SPECT & Fibrosarcoma tumor in rats & 138 \\
\hline $\begin{array}{l}{ }^{111} \text { BnDTPA-NGO- Trastu- } \\
\text { zumab }\end{array}$ & SPECT & Her2 positive tumors in mouse & 139 \\
\hline
\end{tabular}


nano-GO was first reported by the Dai group, where they observed that both GO and nGO-PEG exhibited broad fluorescence emission from the visible region to the IR-A region. ${ }^{121}$ They exploited this IR responsive intrinsic photoluminescence property of GO and synthesized Rituxan (anti-CD20 mAb) conjugated nGO-PEG nanocomposites for targeted fluorescence imaging of CD20 positive Raji B cells using a $658 \mathrm{~nm}$ excitation laser. The main drawback of this study was that the quantum yield of this intrinsic fluorescence of nGO-PEG was very low, that was not suitable for carrying out further in vivo studies. As a result, to overcome this limitation and further improve the fluorescent properties of GO, several different approaches have been carried out which includes loading or linking of organic fluorophores or dyes, inorganic nanomaterials like QDs, upconversion nanoparticles and GQDs onto the surface of GO and rGO sheets. A great deal of attention has been devoted towards the chemistry behind loading and linking these organic and inorganic fluorophores with GO sheets, as direct loading can result in fluorescence quenching of the fluorophore by GO/rGO through energy/charge transfer processes. As a result, spacers such as PEG are used to link these fluorophore moieties to GO/rGO sheets. Yang et al. demonstrated in vivo fluorescence imaging of tumor xenografted mouse models using a NIR dye (Cy7) conjugated nGO-PEG composite. ${ }^{74}$ The authors observed that with time the nGO-PEG-Cy7 nanocomposites accumulated in the tumor regions through passive targeting and showed intense fluorescence signals upon NIR irradiation. In another study, Peng et al. attached fluorescein to PEGylated GO sheets where PEG acted as a spacer between the fluorophore and GO and used it as fluorescent probe for in vitro imaging of HeLa cells (Fig. 6(i)). ${ }^{122}$ Sun et al. reported synthesis of IRDye800-conjugated GO loaded with VEGF as a targeted fluorescent probe for imaging of ischemic muscles in a murine model. ${ }^{123}$ In addition to GO and rGO, another class of graphene-based nanomaterials have gained a great deal of attention in the recent years especially in the field of biomedical imaging. GQDs are nanosized materials in the range of $3-10 \mathrm{~nm}$ having excellent tunable intrinsic photoluminescence or fluorescence properties in the visible region with high biocompatibility, easy surface functionalization and photostable. ${ }^{124}$ Because of these properties, GQDs have emerged as novel biocompatible imaging and diagnostic agents for both in vitro and in vivo applications. Zhu et al. reported the synthesis of biocompatible, water soluble green fluorescent GQDs by a one-step solvothermal process and used it for labeling and imaging of cells in vitro. ${ }^{125}$ In addition to this, there are several reports on synthesis and bioimaging applications of different kinds of GQDs. For a detailed understanding of the bioimaging and theranostic applications of GQDs, readers are requested to refer to the excellent review by Schroeder et al. $^{124}$

\subsection{Two photon fluorescence imaging}

Two photon fluorescence imaging (TPFI) is an improved alternative to single/ one photon fluorescence imaging (OPFI) in the field of bioimaging and diagnostics 


\section{(i) Fluorescence Imaging}
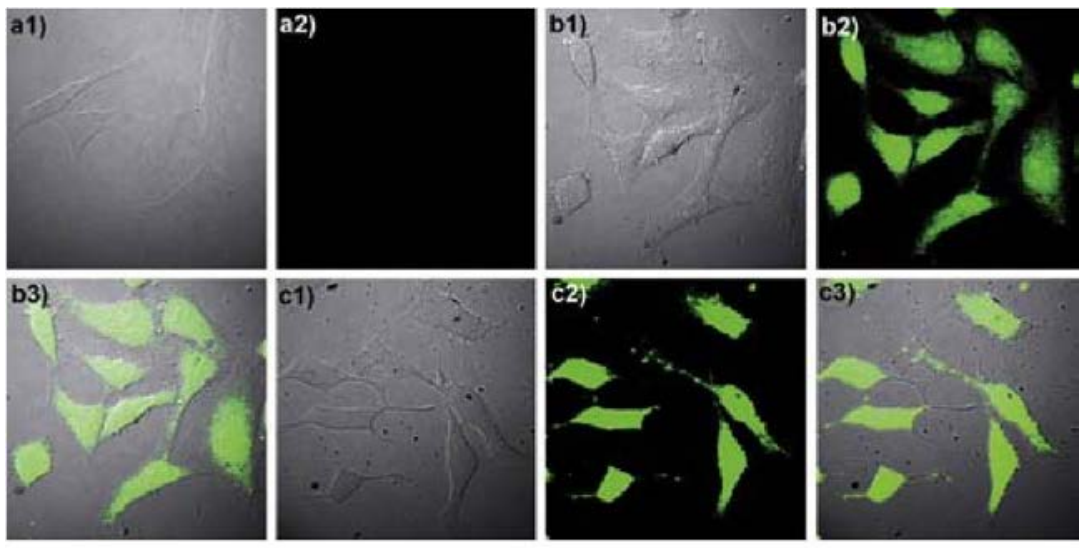

(ii) Raman Imaging
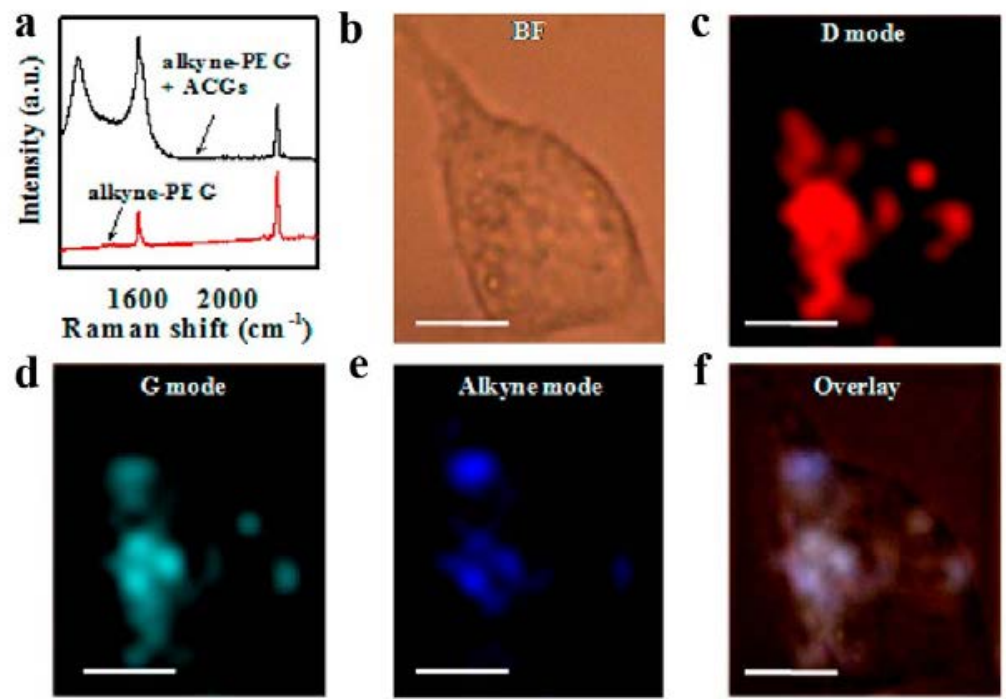

Fig. 6. Graphene-based nanomaterials for bioimaging. (i) Fluorescent imaging. Confocal fluorescence microscopy images of HeLa cells treated with (a) $2.5 \mu \mathrm{g} \mathrm{mL}^{-1}$ PEG-G and Fluo-G suspension for $6 \mathrm{~h}$ at (b) $37^{\circ} \mathrm{C}$ and (c) $4^{\circ} \mathrm{C}$. Bright-field images are marked 1, dark-field images are marked 2, and overlap of images of dark and bright field are marked 3. Reproduced with permission from Ref. 122. Copyright 2010 WileyVCH Verlag GmbH \& Co. KGaA, Weinheim. (ii) Raman imaging. Cell imaging with alkyne-PEG-functionalized ACGs. (a) Raman spectra of alkyne-PEG with (black) and without (red) ACGs. (b)-(f) Raman image of MCF-7 cells treated with alkyne-PEG-modified ACGs using 1s integration/pixel. BF, bright field; scale bar, $10 \mu \mathrm{m}$. Reproduced with permission from Ref. 132. Copyright 2014 American Chemical Society. (iii) Magnetic Resonance imaging. (a) HeLa cells $\left(2 \times 105\right.$ cells mL $\left.{ }^{-1}\right)$ incubated with the $\mathrm{Fe}_{3} \mathrm{O}_{4^{-}}$ GO composites at different concentrations for $24 \mathrm{~h}$. (b) Fe3O4-GO composites $\left(20 \mu \mathrm{g} \mathrm{Fe} \mathrm{mL}^{-1}\right)$ incubated with HeLa cells at different cell densities for $24 \mathrm{~h}$. Reproduced with permission from Ref. 134. Copyright 2011 American Chemical Society. (iv) Radiolabeled imaging. Serial coronal PET images of 4T1 tumorbearing mice at different time points postinjection of ${ }^{64} \mathrm{Cu}-\mathrm{NOTA}-\mathrm{GO}-\mathrm{TRC} 105$, 64Cu-NOTA-GO, or ${ }^{64} \mathrm{Cu}-\mathrm{NOTA}-\mathrm{GO}-\mathrm{TRC} 105$ after a preinjected blocking dose of TRC105. Tumors are indicated by arrowheads. Reproduced with permission from Ref. 136. Copyright 2012 American Chemical Society. 


\section{(iii) Magnetic Resonance Imaging}

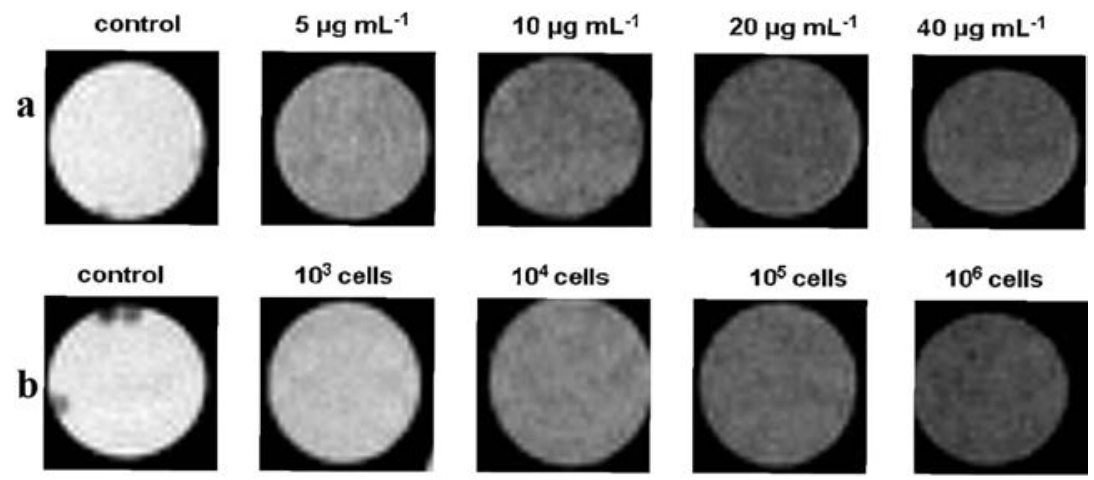

(iv) Radiolabeled Imaging

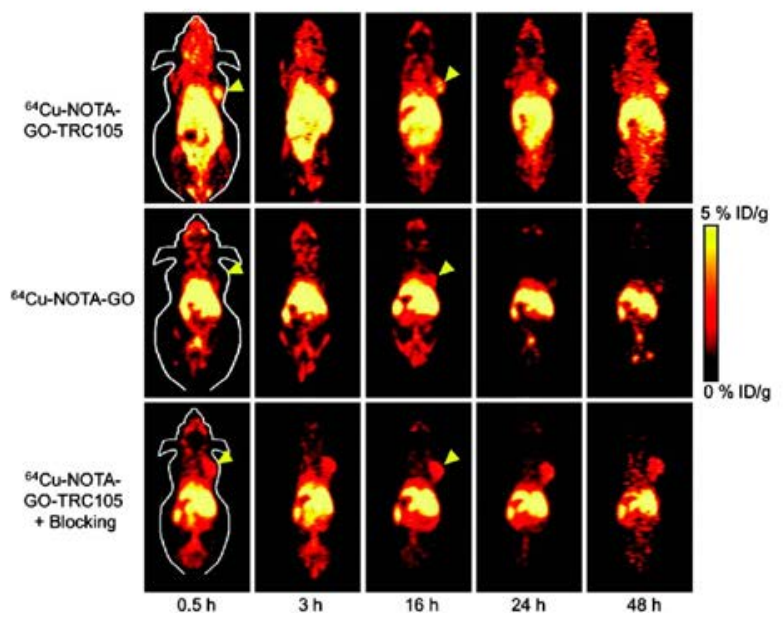

Fig. 6. (Continued)

because of the several advantages that TPFI offers over OPFI such as deeper tissue penetration of NIR, minimal NIR absorbance by surrounding tissues, low autofluorescence and negligible photobleaching. Since TPFI operates in the biologically favorable NIR window, so detailed imaging and investigation at deep tissue level to locate inaccessible solid tumors can be done. Liu et al. synthesized biocompatible nitrogen-doped graphene quantum dots (N-GQDs) and exploited them as optical probes for TPFI of cells and deep tissues. ${ }^{126}$ The authors reported that the N-GQDs could be identified even at a depth of $1800 \mu \mathrm{m}$ in the tissue phantom using TPFI as compared to OPFI which could image the N-GQDs only up to a depth of $400 \mu \mathrm{m}$, thereby demonstrating the advantage and feasibility of graphene-based TPFI for deep tissue imaging. 


\subsection{Raman imaging}

GO exhibits intrinsic Raman signals at $1600 \mathrm{~cm}^{-1}$ ( $\mathrm{G}$ band) and $1350 \mathrm{~cm}^{-1}$ ( $\mathrm{D}$ band), that can be significantly enhanced by using metal nanoparticles like gold or silver to form Raman probes for imaging of cancer cells and tissues. Many studies have been carried out for developing novel graphene-based Raman probes for bioimaging applications. Graphene-based Raman labels have been synthesized both by the in situ reduction of $\mathrm{Ag}^{+}$and $\mathrm{AuCl}_{4}^{-}$on the surface of $\mathrm{GO}$ sheets to form $\mathrm{Ag} / \mathrm{GO}$ and $\mathrm{Au} / \mathrm{GO}$ hybrids, respectively, and also by the direct deposition of $\mathrm{Au}$ and $\mathrm{Ag}$ nanoparticles onto the surface of GO sheets through non-covalent interactions like $\pi-\pi$ stacking and other molecular forces to form $\mathrm{Au} / \mathrm{GO}$ and $\mathrm{Ag} / \mathrm{GO}$ nanocomposites. ${ }^{127-129}$ Liu et al. demonstrated targeted Raman imaging of cancer cells using folic acid conjugated $\mathrm{Ag} / \mathrm{GO}$ hybrids as Raman probes and observed that cells incubated with the hybrids exhibited much stronger Raman signals from the GO sheets as compared to ones incubated with only GO sheets. ${ }^{130}$ Huang et al. studied the cellular uptake mechanisms of GO in the Ca Ski cell line by using Raman probes made up of AuNPs attached to the surface of GO sheets through amide linkages between PEG and DMSA. ${ }^{131}$ Song et al. reported the synthesis of a new type of graphene-based Raman probe for bioimaging. ${ }^{132}$ They synthesized alkyne-PEG functionalized graphitic silver nanoparticles (ACG NPs) using chemical vapor deposition method. Few layers of graphene were grown on the surface of $\mathrm{AgCu}$ NPs by CVD method following which the alkyne-PEG moieties were attached to the surface of GO through non-covalent interactions. These ACG NPs were then used for the Raman imaging of MCF-7 cells, which clearly showed the cellular uptake, localization and distribution of these nanoparticles throughout the cell cytoplasm (Fig. 6(ii)).

\subsection{Magnetic resonance imaging (MRI)}

Magnetic resonance imaging is a widely used imaging and diagnostic tool that allows for non-invasive visualization of organs and tissues in a quantitative manner. Commonly used MRI contrast agents include paramagnetic metals such as gadolinium and manganese which are somewhat toxic to the human body. As a result, to reduce the toxicity and also to enhance the performance of these existing contrast agents, graphene-based nanomaterials have been explored to act as substrates to carry these agents. Gizzatov et al. developed a new type of MRI contrast agent by using carboxyphenylated graphene nanoribbons as substrates to which $\mathrm{Gd}^{3+}$ ions were chelated (Gd-GNR). ${ }^{133}$ It was observed that Gd-GNRs exhibited enhanced MRI contrast and relaxivity as compared to $\mathrm{Gd}^{3+}$-based agents. In another study, Chen et al. reported the synthesis of dextran coated $\mathrm{Fe}_{3} \mathrm{O}_{4} \mathrm{NP}$ loaded GO composites as novel MRI contrast agents for bioimaging (Fig. 6(iii)). ${ }^{134}$ Coating of $\mathrm{Fe}_{3} \mathrm{O}_{4} \mathrm{NPs}$ with dextran allowed formation of aggregates of $\mathrm{Fe}_{3} \mathrm{O}_{4}$ NPs on the surface of GO which in turn helped in enhancing the MRI contrast as compared to single $\mathrm{Fe}_{3} \mathrm{O}_{4}$ NPs. In addition to paramagnetic metals and iron oxide NPs, a non-magnetic carbon 
nanoparticle-based MRI contrast agent was reported for the first time by $\mathrm{Hu}^{135}$ He developed fluorinated GO sheets by creating dipolar $\mathrm{C}-\mathrm{F}$ bonds which acted as the paramagnetic centers and demonstrated good $\mathrm{T}_{2}$ contrast.

\subsection{Radioactive imaging}

Optical imaging techniques such as fluorescence imaging suffers from the limitations of photobleaching, autofluorescence, absorption and scattering of light by surrounding tissues, thereby hampering the quality and contrast of the images. On the other hand, radioactive imaging is a technique that makes use of radioactive substances as labels that can be easily tracked and monitored inside a living system with high sensitivity. The two most commonly used radiolabel imaging techniques are Positron Emission Tomography (PET) and Single-photon emission computed tomography (SPECT). Graphene-based nanomaterials have also been explored as substrates for radiolabel imaging because of the surface properties of graphene that makes functionalization and loading of molecules and imaging agents much easier. Hong et al. developed a novel PET contrast agent by labeling nGO-PEG with ${ }^{64} \mathrm{Cu}$ via a $1,4,7$ - triazacyclononane-1,4,7-triacetic acid (NOTA) linkage. ${ }^{136}$ They further conjugated this nanocomposite with a CD105 specific antibody TRC105 and demonstrated targeted PET imaging of tumor vasculatures in $4 \mathrm{~T} 1$ murine breast tumors (Fig. 6(iv)). In another study by the same group, the researchers developed another PET contrast agent by labeling nGO-PEG with ${ }^{66} \mathrm{Ga}$ and demonstrated tumor targeted PET imaging. ${ }^{137}$ In addition to PET, graphene-based nanomaterials have also been used for developing SPECT imaging agents. Fazaeli et al. developed a SPECT imaging agent by labeling GO nanosheets with ${ }^{198,199} \mathrm{Au}$ and demonstrated targeted SPECT imaging of tumors. ${ }^{138}$ In vivo SPECT imaging of Her2 positive tumors was demonstrated by Cornelissen et al. using ${ }^{111}$ In-benzyl-diethylenetriaminepentaacetic acid (BnDTPA) labelled anti-Her2 antibody conjugated nGO as the imaging agents. ${ }^{139}$ The authors observed that the radiolabeled nanoconstructs showed better pharmacokinetic profiles as compared to radiolabeled antibody alone.

\section{Graphene-Based Theranostic Platforms}

Theranostics refer to diagnostics combined with therapy. Graphene-based nanocomposites have emerged as excellent platforms for theranostic applications in the recent years because of the surface properties of graphene and its derivatives, which allows easy incorporation of multiple modalities into a single platform. Different kinds of multifunctional graphene-based nanotheranostic platforms have been synthesized and exploited for varied biomedical applications by combining imaging techniques with different therapeutic approaches like drug/gene delivery, PTT and PDT. In this section, the theranostic applications of graphene based nanocomposites have been summarized under three main heads- (A) Imaging guided drug/gene 
delivery, (B) Imaging guided phototherapies and (C) Imaging guided chemo-phototherapies, based on the type of therapeutic approach being used. The applications have been further grouped into sections depending upon the type of imaging technique being employed, with the objective of presenting a better categorized appreciation of the immense potential of graphene as nanomedicine. Table 6 summarizes the theranostic applications of graphene-based nanomaterials.

Table 6. Summary of graphene-based nano-theranostic platforms.

\begin{tabular}{|c|c|c|c|c|}
\hline $\begin{array}{l}\text { Therapeutic } \\
\text { modality }\end{array}$ & Imaging modality & $\begin{array}{l}\text { Nano-theranostic } \\
\text { platform }\end{array}$ & $\begin{array}{l}\text { Therapeutic } \\
\text { cargo }\end{array}$ & Reference \\
\hline Drug delivery & Fluorescence & GNC-rGO & DOX & 140 \\
\hline Drug delivery & Fluorescence & Graphene-HQDs-Trf & DOX & 141 \\
\hline Drug delivery & Fluorescence & $\begin{array}{l}\text { cRGD-Chitosan-GO- } \\
\text { Fluorescein }\end{array}$ & DOX & 142 \\
\hline Gene delivery & Fluorescence & BPEI-GO & pDNA & 54 \\
\hline Drug delivery & Raman & $\mathrm{Au} @ \mathrm{NGO}$ & DOX & 143 \\
\hline Drug delivery & Raman & rGO-AuNanostars & DOX & 144 \\
\hline Drug delivery & MRI & GO-DTPA-Gd & DOX & 145 \\
\hline $\begin{array}{l}\text { Drug \& Gene de- } \\
\quad \text { livery }\end{array}$ & MRI & $\mathrm{CMG}$ & DOX, pGFP & 146 \\
\hline $\begin{array}{l}\text { Drug \& Gene de- } \\
\quad \text { livery }\end{array}$ & MRI & PAMAM-Gd-NGO & $\begin{array}{r}\text { Epirubicin, } \\
\text { let-7g } \\
\text { miRNA }\end{array}$ & 147 \\
\hline PTT & Fluorescence & CdSe/ZnS QD-rGO & & 148 \\
\hline PDT & Fluorescence & GO-PEG & DVDMS & 149 \\
\hline PTT \& PDT & UCL & UCNP-nGO & $\mathrm{ZnPc}$ & 150 \\
\hline PDT & Fluorescence & GO-SS-Ce6 & Ce6 & 151 \\
\hline PTT \& PDT & Fluorescence & GO-HA-Ce6 & Ce6 & 152 \\
\hline PTT & Fluorescence & rGONM-PEG-RGD-Cy7 & & 153 \\
\hline PTT & TPFI & GO-PEG-Tf & & 154 \\
\hline PTT \& PDT & Raman & PEG-Au@GON & $\mathrm{ZnPc}$ & 155 \\
\hline PTT & MRI & GO/IONP & & 156 \\
\hline PTT & $\mathrm{PA}$ & ICG-GO-FA & ICG & 157 \\
\hline PTT & PA & BSA-rGO & & 158 \\
\hline PTT & MRI \& CT & $\mathrm{GO} / \mathrm{BaGdF} 5 / \mathrm{PEG}$ & & 159 \\
\hline PTT & MRI \& X-ray & GO-IONP-Au & & 160 \\
\hline PTT & Fluorescence, PA \& MRI & C18PMH-PEG- rGO/IONP & & 161 \\
\hline PTT & Ultrasonic/CT & $\begin{array}{l}\text { Polylactic acid (PLA) micro- } \\
\text { capsules encapsulating GO } \\
\text { and AuNPs }\end{array}$ & & 162 \\
\hline PDT & Fluorescence \& PET & PEG-GO & ${ }^{64} \mathrm{Cu}-\mathrm{HPPH}$ & 163 \\
\hline PTT \& PDT & Fluorescence \& PA & PEG-GO & DVDMS & 164 \\
\hline PTT \& PDT & Fluorescence \& MRI & Magnetic fluorescent graphene & $\mathrm{SiNc}_{4}$ & 165 \\
\hline $\begin{array}{l}\text { Drug delivery \& } \\
\text { PTT }\end{array}$ & Raman \& TPFI & $\begin{array}{l}\text { Graphene-isolated-Au-nano- } \\
\text { crystal (GIAN) }\end{array}$ & DOX & 166 \\
\hline $\begin{array}{l}\text { Drug delivery \& } \\
\text { PTT }\end{array}$ & X-ray & GO@Ag-DOX-NGR & DOX & 167 \\
\hline $\begin{array}{l}\text { Drug delivery \& } \\
\text { PTT }\end{array}$ & MRI & $\begin{array}{l}\text { Magnetic graphene-based } \\
\text { mesoporous silica (MGMS) }\end{array}$ & DOX & 168 \\
\hline $\begin{array}{l}\text { Drug delivery \& } \\
\text { PTT }\end{array}$ & MRI & $\mathrm{FeCo} / \mathrm{GC}$ nanocrystals & DOX & 169 \\
\hline
\end{tabular}




\subsection{Image guided drug/gene delivery}

\subsubsection{Fluorescence imaging-guided drug/gene delivery}

Wang et al. utilized the NIR photoluminescence properties of gold nanoclusters (GNC) for bioimaging applications and loaded them onto rGO sheets to form GNCrGO nanotheranostic platforms for carrying out combined drug delivery and fluorescence imaging of cells in vitro. ${ }^{140}$ Chen et al. developed an imaging probe consisting of QDs coated with hybrid $\mathrm{SiO}_{2}$ shells conjugated to graphene and functionalized with transferrin. ${ }^{141}$ This functionalized nanocomposite was then loaded with doxorubicin and exploited for QD and DOX mediated dual-colored fluorescence imaging-guided tracking and delivery of drug to cancer cells in vitro (Fig. 7(i)). Fluorescein conjugated GO loaded with DOX and coated with cyclicRGD modified chitosan was synthesized by Wang et al. for targeted drug delivery combined with fluorescent imaging of hepatocellular carcinoma cells in vitro. ${ }^{142} \mathrm{Kim}$ et al. synthesized a graphene-based gene delivery vehicle by conjugating low molecular weight branched polyethylenimine (BPEI) to GO sheets, thereby imparting a positive surface charge to the nanoconstruct that ultimately facilitated complexation with negatively charged plasmid DNA through electrostatic interactions and showed efficient gene delivery to HeLa and PC3 cells in vitro. ${ }^{54}$ In addition to this, the authors exploited the intrinsic fluorescence properties of GO for imaging the cellular uptake of the nanoconstructs.

\subsubsection{Raman imaging-guided drug delivery}

Graphene-based Raman probes for theranostic applications have also been developed for combining other therapeutic strategies such as drug delivery with Raman imaging into a single multifunctional platform. Ma et al. reported the synthesis of nanoGO wrapped AuNPs (Au@NGO) as theranostic tools for Raman imaging and drug delivery to HeLa cells. ${ }^{143}$ Similarly, Wang et al. synthesized rGO supported gold nanostars (rGO-NS) as novel sensitive SERS substrates and used them to monitor the loading and release of the drug doxorubicin using SERS. These rGO-NS nanocomposites have the potential to be exploited as a theranostic platform for combined drug delivery and Raman imaging. ${ }^{144}$

\subsubsection{MRI guided-drug/gene delivery}

Zhang et al. developed a graphene-based positive $\mathrm{T}_{1} \mathrm{MRI}$ contrast agent through conjugation of diethylenetriaminepentaacetic acid (DTPA) to GO followed by complexation with gadolinium ions (GO-DTPA-Gd) and successfully used it as a theranostic platform for carrying out MRI imaging combined with delivery of doxorubicin to HepG2 cancer cells in vitro. ${ }^{145}$ The nanocomposite showed improved MRI $\mathrm{T}_{1}$ relaxivity and better cellular MRI contrast as compared to a commercially used contrast agent. Wang et al. combined drug delivery, gene delivery and MRI imaging into a single theranostic platform by synthesizing chitosan functionalized 
(i) Image-Guided Drug Delivery
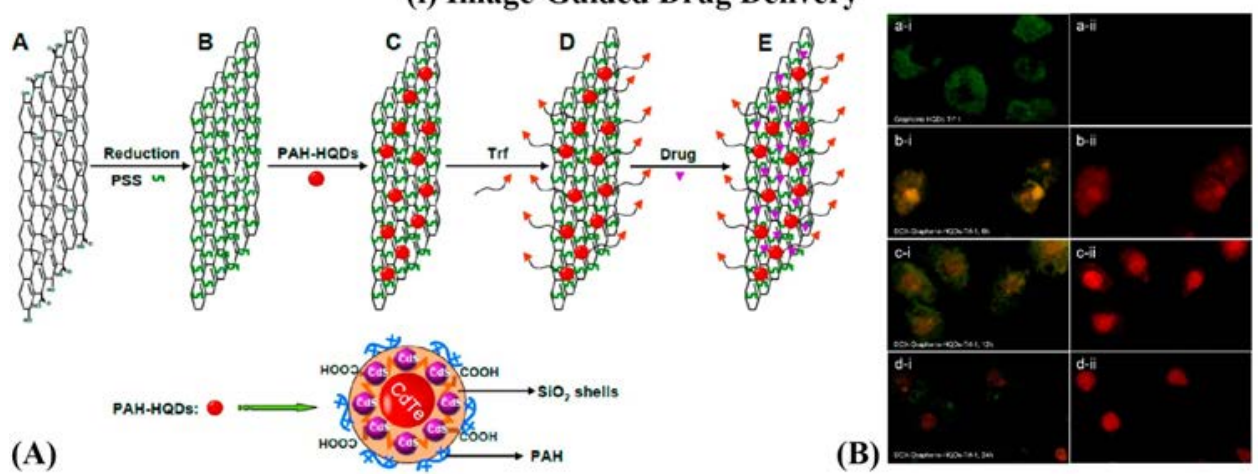

(A)

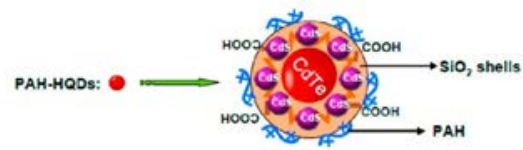

(B)

(ii) Multimodal Image-Guided Phototherapy
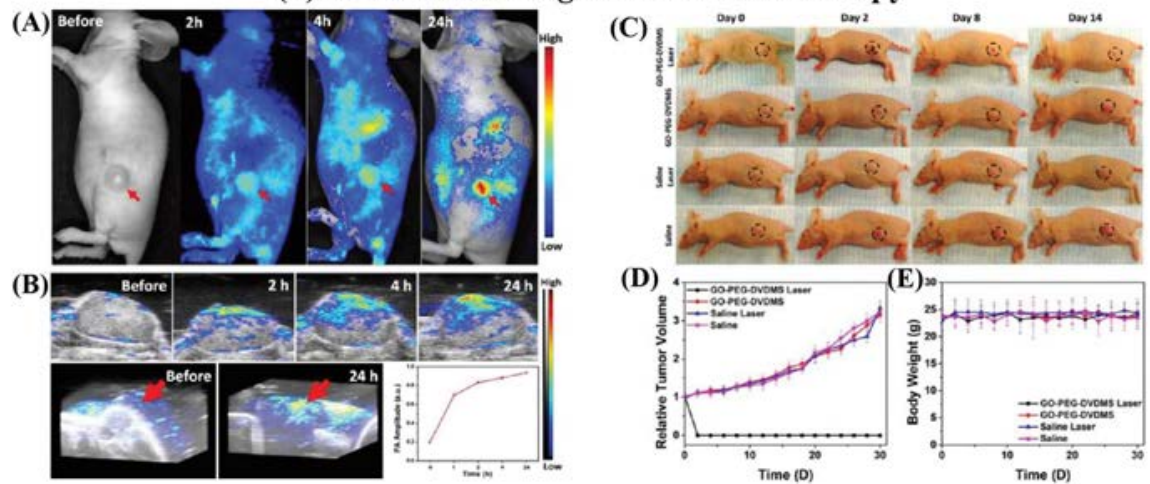

Fig. 7. Graphene-based nanotheranostic platforms. (i) Imaging guided drug delivery. (A) Preparation of Water Dispersible DOX-Graphene-HQDs-Trf (B) Fluorescent microscopic images of HeLa cells directly labeled by graphene-HQDs-Trf-1 for $4 \mathrm{~h}$ (a), and DOX-graphene-HQDs-Trf-1 for $6 \mathrm{~h} \mathrm{(b),} 12 \mathrm{~h}(\mathrm{c})$, and $24 \mathrm{~h}$ (d). ((i), fluorescent images by blue light excitation; (ii), fluorescent images by green light excitation.) Reproduced with permission from Ref. 141. Copyright 2013 American Chemical Society. (ii) Multimodal imaging guided phototherapies. (A) In vivo fluorescence images and (B) ultrasound/photoacoustic images and quantitative analysis of PA signal of PC9 bearing mice treated with GO-PEG-DVDMS (GO-PEG $1 \mathrm{mg} \mathrm{kg}^{-1}$, DVDMS $2 \mathrm{mg} \mathrm{kg}^{-1}$ ) at 2, 4 and $24 \mathrm{~h}$ post-injection. (C) In vivo synergistic therapies of GOPEG-DVDMS. (A) Representative photos of PC9 tumor-bearing mice from different groups after treatment of PDT, PTT or their synergism on day 14. (D) Tumor growth curves of different groups of tumorbearing mice after different treatments. (E) Body weight curves of PC9 tumor mice after different treatments. Reproduced with permission from Ref. 164. Copyright 2015 Royal Society of Chemistry. (iii) Multimodal imaging guided chemo-phototherapy. (A) Schematic illustration of NIR photothermal enhanced chemotherapy mechanism of GIAN/DOX complexes. (B) UV-Vis characterization of the DOXloaded GIANs. Inset: digital photo of the DOX, GIAN, and GIAN/DOX solutions. (C) Fluorescence spectroscopy characterization of the DOX loading efficiency. (D) Cell viability of MCF-7 cells with and without NIR laser irradiation after incubation with free DOX, GIAN, and GIAN/DOX, respectively. (E) Bright field microscopy images of trypan blue-stained MCF-7 cells after different NIR photothermal treatments. Scale bar: $50 \mu \mathrm{m}$. (F) and $(\mathrm{G})$, relative cell viability after treatment with different GIAN concentrations and different $808 \mathrm{~nm}$ laser irradiation times, respectively. Reproduced with permission from Ref. 166. Copyright 2014 Nature publishing group. 
(iii) Multimodal Image-Guided Chemo-Phototherapy
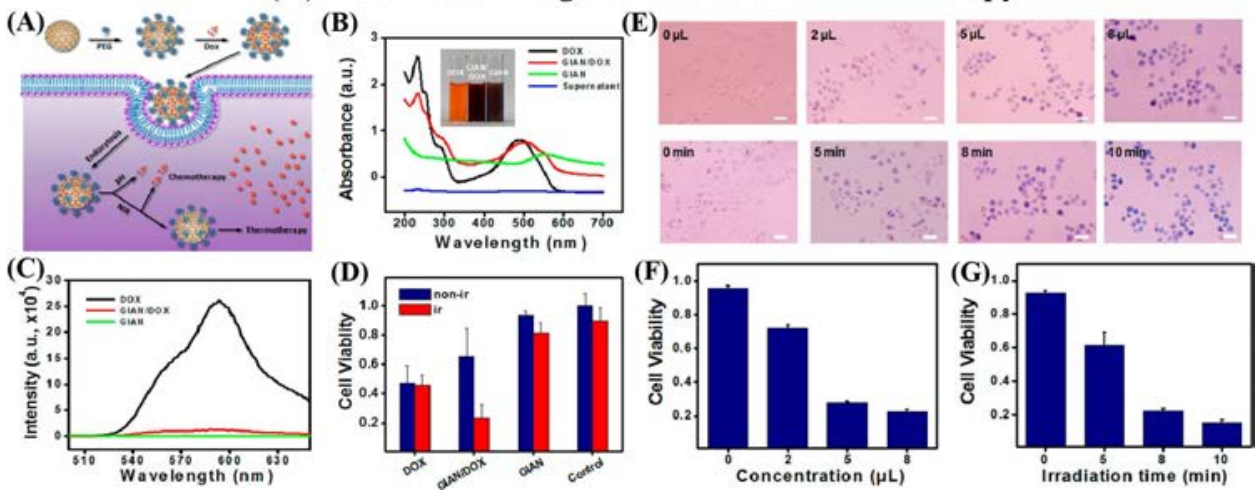

Fig. 7. (Continued)

magnetic graphene nanocomposites (CMG) loaded with the anti-cancer drug DOX and a plasmid DNA encoding GFP and demonstrated efficient delivery of both drug and DNA to A549 lung cancer cells and C42b prostate cancer cells in vitro and also to tumor bearing mice in vivo. ${ }^{146}$ In addition to this, the CMGs acted as strong T2 contrast-enhancing agent thereby enabling MR imaging of the cells and organs. In another study, Yang et al. reported the synthesis of poly (amidoamine) dendrimergrafted gadolinium-functionalized nanographene oxide (Gd-NGO) as theranostic nanocarriers for combined delivery of a chemotherapy drug epirubicin along with Let-7g miRNA. ${ }^{147}$ The Gd-NGO/Let-7g/EPI nanocomposites exhibited higher transfection efficiency and better inhibition of cancer cell growth as compared to chemotherapy or gene therapy alone. The nanocomposites were also used as MRI contrast agents for imaging the integrity of blood-brain barrier and tumor tissue uptake.

\subsection{Imaging-guided phototherapies}

\subsubsection{Fluorescence imaging-guided phototherapy}

Chen et al. exploited the strong fluorescence properties of semiconductor QDs and developed a $\mathrm{CdSe} / \mathrm{ZnS}$ QD labeled, polypeptide functionalized rGO (QD-rGO) nanotheranostic platform for combined fluorescence imaging and photothermal therapy of cancers. ${ }^{148}$ Chen and co-workers demonstrated in vivo PDT of cancer combined with enhanced fluorescence imaging by using PEGylated GO loaded with novel photosensitizer sinoporphyrin sodium (DVDMS) and observed intense fluorescent signals in mice from tumor regions showing enhanced uptake of the nanocomposites by tumors through passive targeting and achieved $100 \%$ tumor elimination in mice intravenously injected with GO-PEG-DVDMS without any loss in body weight and reappearance of tumors. ${ }^{149}$ In another study, Wang et al. 
reported the synthesis of a novel, multifunctional nanotheranostic platform capable of performing synergistically photothermal and photodynamic therapy combined with imaging. ${ }^{150}$ They covalently linked core-shell structured upconversion nanoparticles (UCNP) to the surface of nGO sheets via a PEG spacer, followed by loading a photosensitizer such as phthalocyanine $(\mathrm{ZnPc})$ onto the surface of $\mathrm{nGO}$ to form UCNP-nGO/ZnPc nanocomposites. The researchers successfully demonstrated NIR responsive PTT and PDT combined with UCL based imaging both in vitro and in vivo using this nanocomposite, where, in response to a single light stimulus (NIR) the UCNPs generated upconversion luminescence signals for imaging of cells and whole body animals, nGO converted the NIR light into heat for PTT and ZnPc generated cytotoxic singlet oxygen species for PDT. A redox responsive graphene-based theranostic platform was developed by Cho et al. by conjugating Ce6 to GO nanosheets via redox-responsive cleavable disulfide linker (GO-SS-Ce6). ${ }^{151}$ GO-SS-Ce6 in its native form remains non-fluorescent and non-toxic even in the presence of light irradiation due to quenching by GO. However, when these nanocomposites are taken up by cancer cells, the high concentration of glutathione inside cancer cells results in the cleavage of the disulfide bond, thereby releasing Ce6 into the cells. The released Ce6 molecules regain their fluorescent properties and participate in the generation of ROS upon irradiation with light. In a follow-up study by the same group, the researchers reported the synthesis of an enzyme activatable theranostic agent by forming a non-covalent complex between GO and Hyaluronic acid-Ce6 (HA-Ce6) through physical adsorption of HA-Ce6 on the surface of GO (GO-HA-Ce6). ${ }^{152}$ Hyaluronidase mediated cleavage of GO-HA-Ce6 inside cancer cells resulted in the release of HA-Ce6 with recovery of fluorescence and ROS generation. In addition to this, the GO-HA-Ce6 nanocomposites also exhibited generation of heat due to the NIR responsive properties of GO resulting in stimulus mediated fluorescent imaging guided dual PDT/PTT of cancer cells in vitro. A new class of theranostic platform known as reduced GO nanomesh (rGONM) was developed by Akhavan et al. through the photocatalytic degradation of GO nanoplateletes by using $\mathrm{TiO}_{2}$ nanoparticles. ${ }^{153}$ The rGONMs were functionalized with PEG and conjugated with RGD peptide and cyanine 7 for carrying out targeted fluorescence imaging and photothermal ablation of U87MG glioblastoma tumors in mouse models. The rGONMPEG-RGD-Cy7 nanocomposites showed $100 \%$ elimination of tumors in mice within $48 \mathrm{~h}$ of treatment.

\subsubsection{Two photon fluorescence imaging (TPFI)-guided phototherapy}

Li et al. demonstrated for the first time a theranostic strategy based on TPFI coupled with photothermal therapy using graphene-based nanomaterials. ${ }^{154}$ They synthesized transferrin conjugated GO-PEG nanocomposites as non-bleaching imaging probes for targeted three-dimensional (3D) TPFI of the gastric cancer cell line AGS, using a low power pulsed laser for excitation. In addition to imaging, the authors also observed that the GO nanoparticles could efficiently induce intensive microbubbling 
inside the cells leading to cell death at a laser power as low as $4 \mathrm{~mW}$, owing to the photothermal properties of GO.

\subsubsection{Raman imaging guided-phototherapy}

Kim et al. synthesized a novel theranostic platform for a combined photothermalphotodynamic therapy of cancer guided by Raman imaging. ${ }^{155}$ They synthesized core-shell nanoparticles comprising of an $\mathrm{Au}$ core surrounded by graphene oxide nanocolloid (GON) shell by a one-pot technique, where GONs acted as both reducing agent and stabilizer for the formation of the AuNPs. PEGylation followed by loading of the photosensitizer zinc phthalocyanine $(\mathrm{ZnPc})$ onto these nanoparticles resulted in the formation of biocompatible ZnPc-PEG-Au@GON nano-theranostic platforms, that demonstrated enhanced photo-therapeutic effects on HeLa cells due to the combination of PTT and PDT, and at the same time acted as an efficient Raman probe for in vitro bioimaging of cells.

\subsubsection{MRI guided phototherapy}

MRI guided detection and subsequent photothermal ablation of regional lymph node (RLN) metastasis of pancreatic cancer was demonstrated by Wang et al. using iron oxide nanoparticles (IONP) loaded GO nanocomposites (GO/IONP) as a theranostic agent. ${ }^{156}$ The IONPs helped in providing the MRI contrast which was enhanced due to the presence of GO as a substrate, thereby making RLNs easily distinguishable from the surrounding tissues. The NIR responsive photothermal properties of GO was also enhanced by IONP and was used for the photothermal ablation of the tumors.

\subsubsection{PAI guided phototherapy}

Photoacoustic imaging (PAI) is a non-invasive deep tissue/organ imaging technique that works on the principle of generation of acoustic waves from a sample via absorption of short laser pulses. Biological tissues or organs upon absorbing these short laser pulses tend to generate localized heat that leads to thermal expansion. During contraction, acoustic waves are generated which if recorded by an ultrasonic transducer can result in the formation of an image of the sample by mapping the initial absorbed energy distribution. In this context, RGO have attracted attention as a PA contrast agent because of its better NIR absorbance properties as compared to GO. Wang et al. combined photoacoustic imaging with photothermal therapy into a single platform by loading the NIR absorbing dye indocyanine green (ICG) onto GO and demonstrated targeted photothermal ablation of HeLa cells in vitro alongwith PAI through conjugation of folic acid as the targeting ligand with the ICG-GO nanocomposites. ${ }^{157}$ In vivo PAI alongwith PTT was demonstrated by Sheng et al. using bovine serum albumin functionalized nano-rGO (BSA-rGO) in a mouse bearing MCF-7 xenograft. ${ }^{158}$ Post $2 \mathrm{~h}$ intravenous injection of the BSA-rGO 
nanocomposites, significant photoacoustic signals were observed from the tumor regions showing increased accumulation of nanocomposites in the tumors. The NIR responsive photothermal property of rGO was then used for the photothermal ablation of the tumors.

\subsubsection{Multimodal imaging-guided phototherapy}

Graphene-based nanocomposites capable of performing imaging through multiple modalities such as MRI, CT, fluorescence combined with photo-therapies into a single platform has also been explored. Such systems provide the benefits of better diagnosis by incorporating the advantages of each imaging modality for performing improved and enhanced detection. Zhang et al. demonstrated dual modal MRI/CT imaging of tumors in vivo alongwith photothermal ablation using PEGylated GO nanosheets loaded with BaGdF5 nanoparticles (GO/BaGdF5/ PEG). ${ }^{159}$ The nanocomposites exhibited enhanced magnetic resonance contrast and better X-ray attenuation property than Iohexol which is a standard contrast agent used in radiographic imaging of arteries, veins, ventricles, joints, etc. Intravenous injection of the nanocomposites performed efficient photothermal ablation of the tumors with no toxicity to the host. Shi et al. developed a multifunctional magnetic and plasmonic graphene-based nanocomposite by loading IONP onto the surface of GO sheets followed by growing a layer of gold on it. ${ }^{160}$ This GO-IONP-Au nanocomposite was then functionalized with PEG and used for photothermal ablation of cancer cells and tumors both in vitro and in vivo alongwith dual-modal MRI and X-ray imaging. The gold layer helped in enhancing the NIR absorbance of the nanocomposite and IONP and Au together contributed towards contrast generation for imaging. Yang et al. developed a graphene-based nanotheranostic platform for triple modal fluorescence, photoacoustic, and magnetic resonance imaging of tumors in vivo combined with photothermal therapy and successfully demonstrated multimodal imaging guided photothermal ablation of 4T1-tumors in mice by using C18PMH-PEG functionalized rGO/IONP nanocomposites synthesized via a hydrothermal process. ${ }^{161}$ PLA microcapsules encapsulating GO and AuNPs were developed as nanotheranostic agent by Jin et al. for photothermal ablation of tumors under the guidance of bimodal ultrasonic/CT imaging. ${ }^{162}$ Rong et al. demonstrated bimodal fluorescence/PET imaging guided photodynamic therapy of tumors using PEGylated GO sheets loaded with a radiolabeled photosensitizer ${ }^{64} \mathrm{Cu}-\mathrm{HPPH} .{ }^{163}$ Similarly, in a separate study, Yan et al. demonstrated bimodal fluorescence/PA imaging guided combined photothermal-photodynamic therapy of tumors using PEGylated GO sheets loaded with DVDMS (Fig. 7(ii)). ${ }^{164}$ In another study, Gollavelli et al. developed $\mathrm{SiNc}_{4}$ photosensitizer loaded magnetic fluorescent graphene and demonstrated bimodal fluorescence/MRI imaging guided combined photothermal-photodynamic therapy of HeLa cells in vitro. ${ }^{165}$ 


\subsection{Imaging guided chemo-phototherapies}

Bian et al. reported the synthesis of graphene-isolated-Au-nanocrystal (GIAN) nanostructures by depositing a thin layer of graphene on the surfaces of $\mathrm{Au}$ nanocrystals using CVD method. ${ }^{166}$ These nanostructures were used as imaging probes for dual modal Raman and TPFI imaging of cells and also as a substrate for loading of anti-cancer drug doxorubicin and NIR mediated photothermal therapy of cancer (Fig. 7(iii)). Shi et al. demonstrated tumor targeted chemo-PTT of cancer combined with X-ray imaging of the tumors using GO@Ag nanocomposites. ${ }^{167}$ The GO@Ag nanocomposites were loaded with doxorubicin and conjugated with NGR peptide for active targeting of tumors (GO@Ag-DOX-NGR). The GO@Ag-DOX-NGR showed excellent targeted chemo-photothermal efficacy along with X-ray imaging ability both in in vitro and in vivo studies. Magnetic graphene-based mesoporous silica (MGMS) nanocomposites have emerged as novel theranostic agents for carrying out magnetic imaging-guided therapy. Wang et al. developed a theranostic platform based on magnetic graphene-based mesoporous silica for MRI guided targeted chemo-photothermal therapy of gliomas. ${ }^{168}$ The MGMS nanocomposites were tagged with a IL-13 targeting peptide and loaded with DOX for targeted delivery of the drugs to U251 glioma cells. The magnetic properties of the nanocomposite due to the presence of IONPs on the surface of GO sheets provided improved contrast for MR imaging as seen from increased $\mathrm{T}_{2}$ relaxation rates and at the same time enhanced the optical absorbance of the nanocomposites in the NIR region thereby making them suitable for photothermal applications also. Sherlock et al. reported a new type of theranostic platform by synthesizing ultrasmall FeCo/graphitic carbon shell nanocrystals $\left(\mathrm{FeCo} / \mathrm{GC}\right.$ ) having size in the range of $3-4 \mathrm{~nm}$ in diameter. ${ }^{169}$ The $\mathrm{FeCo} / \mathrm{GC}$ nanocrystals exhibited excellent magnetization, high doxorubicin loading efficiency and both $\mathrm{pH}$ and NIR responsive drug release abilities that resulted in enhanced cytotoxicity towards cancer cells. NIR induced hyperthermia also increased the cellular uptake of these nanocrystals thereby further enhancing the cytotoxic effects. In addition to this, the nanocrystals were also used as MRI contrast agents for imaging their cellular uptake.

\section{Conclusion and Outlook}

In this review, we provide a systematic and detailed overview regarding the current progress on the different biomedical applications of graphene-based nanomaterials in the field of drug delivery, gene delivery, phototherapy, bioimaging and combined therapeutic and theranostic approaches in a highly categorized manner. The versatility of graphene and its derivatives in the field of biomedical research can be attributed to the unique structural, optical, electrical, thermal and biocompatible properties of these nanomaterials. The monolayer single-atomic $2 \mathrm{D}$ nanosheets of graphene oxide and rGO provide ultra-high surface area and numerous functional groups on their surface for highly efficient loading of different kinds of therapeutic 
and theranostic cargo such as anti-cancer drugs, nucleic acids like DNA, RNA, siRNA, miRNA, imaging agents like fluorophores, quantum dots, dyes, inorganic materials like plasmonic and magnetic nanoparticles, etc., through hydrophobic and $\pi-\pi$ interactions. It also allows easy functionalization with biocompatible polymers like PEG and targeting moieties like peptides, vitamins and antibodies through covalent and non-covalent interactions for the development of novel multifunctional biocompatible theranostic platforms for imaging guided therapy. This ability to combine multiple treatment and diagnostic modalities into a single platform have significantly increased the therapeutic efficiency and importance of graphene in the field of nanomedicine and has made it a material of choice for exploring different novel biological applications. However, in spite of this tremendous progress, most of the research that has been carried out so far on graphene and its derivatives have mainly explored its therapeutic potential at the in vitro level, with very little focus on understanding the interactions of graphene with cells and tissues at the organismal level, which is very crucial for a successful translation of a graphene-based nanomedicine from bench to bedside. Detailed mechanistic studies in small as well as large animal models should be done to get a clear understanding of how graphene-based nanomaterials behave once they are inside the body of a host, how they interact with the cells of the immune system and reticuloendothelial system, whether they induce any local or systemic inflammatory response, their pharmacokinetics and biodistribution profiles and most importantly, their toxicity profiles. Some studies have shown that the toxicity and interaction of graphene-based materials with cells and tissues are greatly affected by the size distribution of these materials, with larger size having more toxicity and higher chances of getting phagocytosed and cleared off the circulation by phagocytic cells. However, the large size distribution of these materials that arise in each batch during their synthesis makes it very difficult to correlate size with toxicity and hence requires the development of new synthesis methods that will provide uniformity in the size of these nanoparticles. In addition to synthesis, new surface functionalization strategies for reducing toxicity and improving physiological stability should also be developed. So, by looking at the progress that have been made in the field of graphene research over the years, we can surely say that graphene holds a lot of promise and hope as a future nanomedicine. However, to take it to the clinic a significant amount of effort from scientists and researchers from different disciplines of chemistry, material science, engineering and specially biology is needed to understand the systemic toxicity and immunogenicity of the material.

\section{Acronym list}

GO - Graphene oxide

rGO - Reduced graphene oxide

GQD - Graphene quantum dot

NIR — Near infrared 


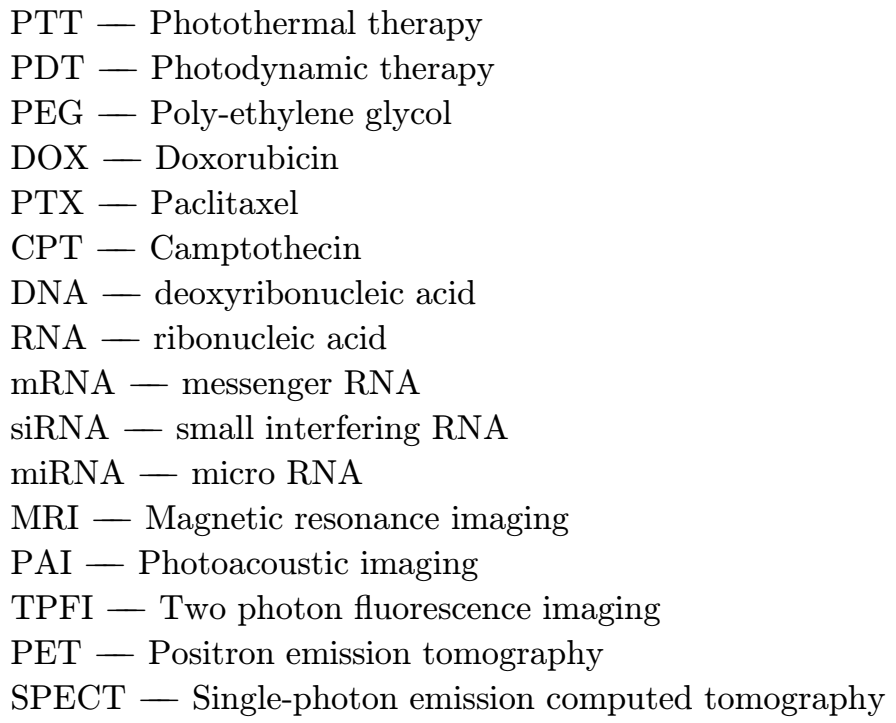

\section{Acknowledgment}

The authors would like to acknowledge the financial and infrastructural support provided by BioX Centre, Indian Institute of Technology Mandi. SR would like to acknowledge DST INSPIRE Fellowship programme [IF160513] for providing doctoral fellowship.

\section{References}

1. A. K. Geim and K. S. Novoselov, The rise of graphene, Nat. Mater. 6 (2007) 183-191.

2. K. S. Novoselov, A. K. Geim, S. V. Morozov, D. Jiang, Y. Zhang, S. V. Dubonos et al., Electric field effect in atomically thin carbon films, Science 306 (2004) 666-669.

3. A. K. Geim, Graphene: Status and prospects, Science 324 (2009) 1530-1534.

4. K. S. Novoselov, V. Fal, L. Colombo, P. Gellert, M. Schwab and K. Kim, A roadmap for graphene, Nature 490 (2012) 192-200.

5. D. R. Dreyer, S. Park, C. W. Bielawski and R. S. Ruoff, The chemistry of graphene oxide, Chem. Soc. Rev. 39 (2010) 228-240.

6. R. S. Edwards and K. S. Coleman, Graphene synthesis: Relationship to applications, Nanoscale 5 (2013) 38-51.

7. P. Suvarnaphaet and S. Pechprasarn, Graphene-Based Materials for Biosensors: A Review, Sensors 17 (2017) 2161.

8. V. Georgakilas, M. Otyepka, A. B. Bourlinos, V. Chandra, N. Kim, K. C. Kemp et al., Functionalization of graphene: Covalent and non-covalent approaches, derivatives and applications, Chem. Rev 112 (2012) 6156-6214.

9. K. Yang, L. Feng, H. Hong, W. Cai and Z. Liu, Preparation and functionalization of graphene nanocomposites for biomedical applications, Nat. Protocols 8 (2013).

10. S. C. Patel, S. Lee, G. Lalwani, C. Suhrland, S. M. Chowdhury and B. Sitharaman, Graphene-based platforms for cancer therapeutics, Therap. Deliv. 7 (2016) 101-116. 
11. S. Goenka, V. Sant and S. Sant, Graphene-based nanomaterials for drug delivery and tissue engineering, J. Control. Release 173 (2014) 75-88.

12. M. Orecchioni, R. Cabizza, A. Bianco and L. G. Delogu, Graphene as cancer theranostic tool: Progress and future challenges, Theranostics 5 (2015) 710.

13. S.-Y. Wu, S. S. A. An and J. Hulme, Current applications of graphene oxide in nanomedicine, Int. J. Nanomed. 10 (2015) 9.

14. M. Nurunnabi, K. Parvez, M. Nafiujjaman, V. Revuri, H. A. Khan, X. Feng et al., Bioapplication of graphene oxide derivatives: Drug/gene delivery, imaging, polymeric modification, toxicology, therapeutics and challenges, RSC Adv. 5 (2015) 42141-42161.

15. K. Yang, L. Feng, X. Shi and Z. Liu, Nano-graphene in biomedicine: Theranostic applications, Chem. Soc. Rev. 42 (2013) 530-547.

16. Z. Liu, J. T. Robinson, X. Sun and H. Dai, PEGylated nanographene oxide for delivery of water-insoluble cancer drugs, J. Am. Chem. Soc. 130 (2008) 10876-10877.

17. X. Yang, X. Zhang, Z. Liu, Y. Ma, Y. Huang and Y. Chen, High-efficiency loading and controlled release of doxorubicin hydrochloride on graphene oxide, J. Phys. Chem. C 112 (2008) 17554-17558.

18. L. Zhang, J. Xia, Q. Zhao, L. Liu and Z. Zhang, Functional graphene oxide as a nanocarrier for controlled loading and targeted delivery of mixed anticancer drugs, Small 6 (2010) 537-544.

19. T. L. Moore, R. Podilakrishna, A. Rao and F. Alexis, Systemic administration of polymer-coated nano-graphene to deliver drugs to glioblastoma, Part. Part. Syst. Char. 31 (2014) 886-894.

20. Z. Xu, S. Zhu, M. Wang, Y. Li, P. Shi and X. Huang, Delivery of paclitaxel using PEGylated graphene oxide as a nanocarrier, ACS Appl. Mater. Interfaces 7 (2015) 1355-1363.

21. A. Angelopoulou, E. Voulgari, E. Diamanti, D. Gournis and K. Avgoustakis, Graphene oxide stabilized by PLA-PEG copolymers for the controlled delivery of paclitaxel, Eur. J. Pharmaceut. Biopharmaceut. 93 (2015) 18-26.

22. Q. Lin, X. Huang, J. Tang, Y. Han and H. Chen, Environmentally friendly, one-pot synthesis of folic acid-decorated graphene oxide-based drug delivery system, J. Nanopart. Res. 15 (2013) 2144.

23. W. Miao, G. Shim, C. M. Kang, S. Lee, Y. S. Choe, H.-G. Choi et al., Cholesteryl hyaluronic acid-coated, reduced graphene oxide nanosheets for anti-cancer drug delivery, Biomaterials 34 (2013) 9638-9647.

24. H. Wu, H. Shi, Y. Wang, X. Jia, C. Tang, J. Zhang et al., Hyaluronic acid conjugated graphene oxide for targeted drug delivery, Carbon 69 (2014) 379-389.

25. G. Liu, H. Shen, J. Mao, L. Zhang, Z. Jiang, T. Sun et al., Transferrin modified graphene oxide for glioma-targeted drug delivery: In vitro and in vivo evaluations, ACS Appl. Mater. Interfaces 5 (2013) 6909-6914.

26. H. Wang, W. Gu, N. Xiao, L. Ye and Q. Xu, Chlorotoxin-conjugated graphene oxide for targeted delivery of an anticancer drug, Int. J. Nanomed. 9 (2014) 1433.

27. S. M. Chowdhury, G. Lalwani, K. Zhang, J. Y. Yang, K. Neville and B. Sitharaman, Cell specific cytotoxicity and uptake of graphene nanoribbons, Biomaterials 34 (2013) 283-293.

28. S. M. Chowdhury, P. Manepalli and B. Sitharaman, Graphene nanoribbons elicit cell specific uptake and delivery via activation of epidermal growth factor receptor enhanced by human papillomavirus E5 protein, Acta Biomater. 10 (2014) 4494-4504.

29. S. M. Chowdhury, C. Surhland, Z. Sanchez, P. Chaudhary, M. S. Kumar, S. Lee et al., Graphene nanoribbons as a drug delivery agent for lucanthone mediated therapy of glioblastoma multiforme, Nanomed. Nanotechnol. Biol. Med. 11 (2015) 109-118. 
30. H. Bai, C. Li, X. Wang and G. Shi, A pH-sensitive graphene oxide composite hydrogel, Chem. Commun. 46 (2010) 2376-2378.

31. D. Depan, J. Shah and R. Misra, Controlled release of drug from folate-decorated and graphene mediated drug delivery system: Synthesis, loading efficiency and drug release response, Mater. Sci. Eng. C 31 (2011) 1305-1312.

32. M. Kakran, N. G. Sahoo, H. Bao, Y. Pan and L. Li, Functionalized graphene oxide as nanocarrier for loading and delivery of ellagic acid, Current Med. Chem. 18 (2011) 4503-4512.

33. J. Wu, Y.-S. Wang, X.-Y. Yang, Y.-Y. Liu, J.-R. Yang, R. Yang et al., Graphene oxide used as a carrier for adriamycin can reverse drug resistance in breast cancer cells, Nanotechnology 23 (2012) 355101.

34. J. Liu, S. Guo, L. Han, T. Wang, W. Hong, Y. Liu et al., Synthesis of phospholipid monolayer membrane functionalized graphene for drug delivery, J. Mater. Chem. 22 (2012) 20634-20640.

35. X. Fan, G. Jiao, L. Gao, P. Jin and X. Li, The preparation and drug delivery of a graphene-carbon nanotube-Fe 3 O 4 nanoparticle hybrid, J. Mater. Chem. B 1 (2013) $2658-2664$.

36. E. Song, W. Han, C. Li, D. Cheng, L. Li, L. Liu et al., Hyaluronic acid-decorated graphene oxide nanohybrids as nanocarriers for targeted and $\mathrm{pH}$-responsive anticancer drug delivery, ACS Appl. Mater. Interfaces 6 (2014) 11882-11890.

37. S. Jokar, A. Pourjavadi and M. Adeli, Albumin-graphene oxide conjugates; carriers for anticancer drugs, Rsc Adv. 4 (2014) 33001-33006.

38. G. Wei, R. Dong, D. Wang, L. Feng, S. Dong, A. Song et al., Functional materials from the covalent modification of reduced graphene oxide and $\beta$-cyclodextrin as a drug delivery carrier, New J. Chem. 38 (2014) 140-145.

39. T. Zhou, X. Zhou and D. Xing, Controlled release of doxorubicin from graphene oxide based charge-reversal nanocarrier, Biomaterials 35 (2014) 4185-4194.

40. H. Wen, C. Dong, H. Dong, A. Shen, W. Xia, X. Cai et al., Engineered redox-responsive PEG detachment mechanism in PEGylated nano-graphene oxide for intracellular drug delivery, Small 8 (2012) 760-769.

41. U. Dembereldorj, M. Kim, S. Kim, E.-O. Ganbold, S. Y. Lee and S.-W. Joo, A spatiotemporal anticancer drug release platform of PEGylated graphene oxide triggered by glutathione in vitro and in vivo, J. Mater. Chem. 22 (2012) 23845-23851.

42. H. Kim, D. Lee, J. Kim, T.-i. Kim and W. J. Kim, Photothermally triggered cytosolic drug delivery via endosome disruption using a functionalized reduced graphene oxide, ACS Nano 7 (2013) 6735-6746.

43. K. Zhou, Y. Zhu, X. Yang and C. Li, One-pot preparation of graphene/Fe $3 \mathrm{O} 4 \mathrm{com}-$ posites by a solvothermal reaction, New J. Chem. 34 (2010) 2950-2955.

44. X. Li, X. Huang, D. Liu, X. Wang, S. Song, L. Zhou et al., Synthesis of 3D hierarchical Fe3O4/graphene composites with high lithium storage capacity and for controlled drug delivery, J. Phys. Chem. C 115 (2011) 21567-21573.

45. H.-W. Liu, S.-H. Hu, Y.-W. Chen and S.-Y. Chen, Characterization and drug release behavior of highly responsive chip-like electrically modulated reduced graphene oxide-poly (vinyl alcohol) membranes, J. Mater. Chem. 22 (2012) 17311-17320.

46. C. E. Thomas, A. Ehrhardt and M. A. Kay, Progress and problems with the use of viral vectors for gene therapy, Nat. Rev. Genet. 4 (2003) 346.

47. H. Yin, R. L. Kanasty, A. A. Eltoukhy, A. J. Vegas, J. R. Dorkin and D. G. Anderson, Non-viral vectors for gene-based therapy, Nat. Rev. Genet. 15 (2014) 541.

48. M. Vincent, I. de Lázaro and K. Kostarelos, Graphene materials as 2D non-viral gene transfer vector platforms, Gene Therapy 24 (2017) 123-132. 
49. C.-H. Lu, C.-L. Zhu, J. Li, J.-J. Liu, X. Chen and H.-H. Yang, Using graphene to protect DNA from cleavage during cellular delivery, Chem. Commun. 46 (2010) 3116-3118.

50. Y. Wang, Z. Li, D. Hu, C.-T. Lin, J. Li and Y. Lin, Aptamer/graphene oxide nanocomplex for in situ molecular probing in living cells, J. Am. Chem. Soc. 132 (2010) 9274-9276.

51. H. Dong, L. Ding, F. Yan, H. Ji and H. Ju, The use of polyethylenimine-grafted graphene nanoribbon for cellular delivery of locked nucleic acid modified molecular beacon for recognition of microRNA, Biomaterials 32 (2011) 3875-3882.

52. H. Dong, W. Dai, H. Ju, H. Lu, S. Wang, L. Xu et al., Multifunctional poly (l-lactide)polyethylene glycol-grafted graphene quantum dots for intracellular microRNA imaging and combined specific-gene-targeting agents delivery for improved therapeutics, ACS Appl. Mater. Interfaces 7 (2015) 11015-11023.

53. B. Chen, M. Liu, L. Zhang, J. Huang, J. Yao and Z. Zhang, Polyethylenimine-functionalized graphene oxide as an efficient gene delivery vector, J. Mater. Chem. 21 (2011) $7736-7741$.

54. H. Kim, R. Namgung, K. Singha, I.-K. Oh and W. J. Kim, Graphene oxide-polyethylenimine nanoconstruct as a gene delivery vector and bioimaging tool, Bioconjugate Chem. 22 (2011) 2558-2567.

55. T. Ren, L. Li, X. Cai, H. Dong, S. Liu and Y. Li, Engineered polyethylenimine/graphene oxide nanocomposite for nuclear localized gene delivery, Polymer Chem. 3 (2012) 25612569.

56. X. Zhou, F. Laroche, G. E. Lamers, V. Torraca, P. Voskamp, T. Lu et al., Ultra-small graphene oxide functionalized with polyethylenimine (PEI) for very efficient gene delivery in cell and zebrafish embryos, Nano Res. (2012) 1-7.

57. L. Feng, S. Zhang and Z. Liu, Graphene based gene transfection, Nanoscale 3 (2011) 1252-1257.

58. C. Xu, D. Yang, L. Mei, B. Lu, L. Chen, Q. Li et al., Encapsulating gold nanoparticles or nanorods in graphene oxide shells as a novel gene vector, ACS Appl. Mater. Interfaces 5 (2013) 2715-2724.

59. L. Feng, X. Yang, X. Shi, X. Tan, R. Peng, J. Wang et al., Polyethylene glycol and polyethylenimine dual-functionalized nano-graphene oxide for photothermally enhanced gene delivery, Small 9 (2013) 1989-1997.

60. H. Kim and W. J. Kim, Photothermally controlled gene delivery by reduced graphene oxide-polyethylenimine nanocomposite, Small 10 (2014) 117-126.

61. K. Li, L. Feng, J. Shen, Q. Zhang, Z. Liu, S.-T. Lee et al., Patterned substrates of nanographene oxide mediating highly localized and efficient gene delivery, ACS Appl. Mater. Interfaces 6 (2014) 5900-5907.

62. A. Paul, A. Hasan, H. A. Kindi, A. K. Gaharwar, V. T. Rao, M. Nikkhah et al., Injectable graphene oxide/hydrogel-based angiogenic gene delivery system for vasculogenesis and cardiac repair, ACS Nano 8 (2014) 8050-8062.

63. H. Y. Choi, T.-J. Lee, G.-M. Yang, J. Oh, J. Won, J. Han et al., Efficient mRNA delivery with graphene oxide-polyethylenimine for generation of footprint-free human induced pluripotent stem cells, J. Controlled Rel. 235 (2016) 222-235.

64. S. Baek, J. Oh, J. Song, H. Choi, J. Yoo, G. Y. Park et al., Generation of IntegrationFree Induced Neurons Using Graphene Oxide-Polyethylenimine, Small 13 (2017).

65. M. Y. Chiang, Y. Z. Lin, S. J. Chang, W. C. Shyu, H. E. Lu and S. Y. Chen, Direct reprogramming of human suspension cells into mesodermal cell lineages via combined magnetic targeting and photothermal stimulation by magnetic graphene oxide complexes, Small (2017). 
66. H. Bao, Y. Pan, Y. Ping, N. G. Sahoo, T. Wu, L. Li et al., Chitosanfunctionalized graphene oxide as a nanocarrier for drug and gene delivery, Small 7 (2011) 1569-1578.

67. X. Liu, D. Ma, H. Tang, L. Tan, Q. Xie, Y. Zhang et al., Polyamidoamine dendrimer and oleic acid-functionalized graphene as biocompatible and efficient gene delivery vectors, ACS Appl. Mater. Interfaces 6 (2014) 8173-8183.

68. S. K. Tripathi, R. Goyal, K. C. Gupta and P. Kumar, Functionalized graphene oxide mediated nucleic acid delivery, Carbon 51 (2013) 224-235.

69. Y.-P. Huang, C.-M. Hung, Y.-C. Hsu, C.-Y. Zhong, W.-R. Wang, C.-C. Chang et al., Suppression of breast cancer cell migration by small interfering RNA delivered by polyethylenimine-functionalized graphene oxide, Nanoscale Res. Lett. 11 (2016) 247.

70. R. Imani, W. Shao, S. Taherkhani, S. H. Emami, S. Prakash and S. Faghihi, Dual-functionalized graphene oxide for enhanced siRNA delivery to breast cancer cells, Colloids Surf. B, Biointerfaces 147 (2016) 315-325.

71. V. Shanmugam, S. Selvakumar and C.-S. Yeh, Near-infrared light-responsive nanomaterials in cancer therapeutics, Chem. Soc. Rev. 43 (2014) 6254-6287.

72. Y.-W. Chen, Y.-L. Su, S.-H. Hu and S.-Y. Chen, Functionalized graphene nanocomposites for enhancing photothermal therapy in tumor treatment, Adv. Drug Deliv. Rev. 105 (2016) 190-204.

73. Y. Li, H. Dong, Y. Li and D. Shi, Graphene-based nanovehicles for photodynamic medical therapy, Int. J. Nanomed. 10 (2015) 2451.

74. K. Yang, S. Zhang, G. Zhang, X. Sun, S.-T. Lee and Z. Liu, Graphene in mice: Ultrahigh in vivo tumor uptake and efficient photothermal therapy, Nano Lett. 10 (2010) 3318-3323.

75. J. T. Robinson, S. M. Tabakman, Y. Liang, H. Wang, H. Sanchez Casalongue, D. Vinh et al., Ultrasmall reduced graphene oxide with high near-infrared absorbance for photothermal therapy, J. Am. Chem. Soc. 133 (2011) 6825-6831.

76. K. Yang, J. Wan, S. Zhang, B. Tian, Y. Zhang and Z. Liu, The influence of surface chemistry and size of nanoscale graphene oxide on photothermal therapy of cancer using ultra-low laser power, Biomaterials 33 (2012) 2206-2214.

77. O. Akhavan, E. Ghaderi, S. Aghayee, Y. Fereydooni and A. Talebi, The use of a glucosereduced graphene oxide suspension for photothermal cancer therapy, J. Mater. Chem. 22 (2012) 13773-13781.

78. M. Li, X. Yang, J. Ren, K. Qu and X. Qu, Using graphene oxide high near-infrared absorbance for photothermal treatment of Alzheimer's disease, Adv. Mater. 24 (2012) 1722-1728.

79. M. Abdolahad, M. Janmaleki, S. Mohajerzadeh, O. Akhavan and S. Abbasi, Polyphenols attached graphene nanosheets for high efficiency NIR mediated photodestruction of cancer cells, Mater. Sci. Eng. C 33 (2013) 1498-1505.

80. C. Hou, H. Quan, Y. Duan, Q. Zhang, H. Wang and Y. Li, Facile synthesis of waterdispersible $\mathrm{Cu} 2 \mathrm{O}$ nanocrystal-reduced graphene oxide hybrid as a promising cancer therapeutic agent, Nanoscale 5 (2013) 1227-1232.

81. D.-K. Lim, A. Barhoumi, R. G. Wylie, G. Reznor, R. S. Langer and D. S. Kohane, Enhanced photothermal effect of plasmonic nanoparticles coated with reduced graphene oxide, Nano Lett. 13 (2013) 4075-4079.

82. U. Dembereldorj, S. Y. Choi, E. O. Ganbold, N. W. Song, D. Kim, J. Choo et al., Gold nanorod-assembled PEGylated graphene-oxide nanocomposites for photothermal cancer therapy, Photochem. Photobiol. 90 (2014) 659-666. 
83. J. L. Li, X. L. Hou, H. C. Bao, L. Sun, B. Tang, J. F. Wang et al., Graphene oxide nanoparticles for enhanced photothermal cancer cell therapy under the irradiation of a femtosecond laser beam, J. Biomed. Mater. Res. A 102 (2014) 2181-2188.

84. H. S. Jung, W. H. Kong, D. K. Sung, M.-Y. Lee, S. E. Beack, D. H. Keum et al., Nanographene oxide-hyaluronic acid conjugate for photothermal ablation therapy of skin cancer, ACS Nano 8 (2014) 260-268.

85. O. Akhavan, A. Meidanchi, E. Ghaderi and S. Khoei, Zinc ferrite spinel-graphene in magneto-photothermal therapy of cancer, J. Mater. Chem. B 2 (2014) 3306-3314.

86. L. Yang, Y.-T. Tseng, G. Suo, L. Chen, J. Yu, W.-J. Chiu et al., Photothermal therapeutic response of cancer cells to aptamer-gold nanoparticle-hybridized graphene oxide under NIR illumination, ACS Appl. Mater. Interfaces 7 (2015) 5097-5106.

87. M. Guo, J. Huang, Y. Deng, H. Shen, Y. Ma, M. Zhang et al., pH-responsive cyaninegrafted graphene oxide for fluorescence resonance energy transfer-enhanced photothermal therapy, Adv. Function. Mater. 25 (2015) 59-67.

88. H. Dong, Z. Zhao, H. Wen, F. Guo, A. Shen, F. Pilger et al., Poly (ethylene glycol) conjugated nano-graphene oxide for photodynamic therapy, Sci. China Chem. $\mathbf{5 3}$ (2010) 2265-2271.

89. P. Huang, C. Xu, J. Lin, C. Wang, X. Wang, C. Zhang et al., Folic acid-conjugated graphene oxide loaded with photosensitizers for targeting photodynamic therapy, Theranostics 1 (2011) 240.

90. L. Zhou, H. Jiang, S. Wei, X. Ge, J. Zhou and J. Shen, High-efficiency loading of hypocrellin B on graphene oxide for photodynamic therapy, Carbon 50 (2012) 55945604 .

91. L. Zhou, W. Wang, J. Tang, J. H. Zhou, H. J. Jiang and J. Shen, Graphene oxide noncovalent photosensitizer and its anticancer activity in vitro, Chem. A Eur. J. 17 (2011) 12084-12091.

92. Z. Hu, Y. Huang, S. Sun, W. Guan, Y. Yao, P. Tang et al., Visible light driven photodynamic anticancer activity of graphene oxide/TiO 2 hybrid, Carbon 50 (2012) 994-1004.

93. Z. Hu, J. Li, C. Li, S. Zhao, N. Li, Y. Wang et al., Folic acid-conjugated graphene-ZnO nanohybrid for targeting photodynamic therapy under visible light irradiation, J. Mater. Chem. B 1 (2013) 5003-5013.

94. F. Li, S.-J. Park, D. Ling, W. Park, J. Y. Han, K. Na et al., Hyaluronic acid-conjugated graphene oxide/photosensitizer nanohybrids for cancer targeted photodynamic therapy, J. Mater. Chem. B 1 (2013) 1678-1686.

95. M. Wojtoniszak, D. Rogińska, B. Machaliński, M. Drozdzik and E. Mijowska, Graphene oxide functionalized with methylene blue and its performance in singlet oxygen generation, Mater. Res. Bull. 48 (2013) 2636-2639.

96. W. Zhang, Z. Guo, D. Huang, Z. Liu, X. Guo and H. Zhong, Synergistic effect of chemo-photothermal therapy using PEGylated graphene oxide, Biomaterials 32 (2011) $8555-8561$.

97. X. Qin, Z. Guo, Z. Liu, W. Zhang, M. Wan and B. Yang, Folic acid-conjugated graphene oxide for cancer targeted chemo-photothermal therapy, J. Photochem. Photobiol. B: Biol. 120 (2013) 156-162.

98. H.-W. Yang, Y.-J. Lu, K.-J. Lin, S.-C. Hsu, C.-Y. Huang, S.-H. She et al., EGRF conjugated PEGylated nanographene oxide for targeted chemotherapy and photothermal therapy, Biomaterials 34 (2013) 7204-7214.

99. C. Xu, D. Yang, L. Mei, Q. Li, H. Zhu and T. Wang, Targeting chemophotothermal therapy of hepatoma by gold nanorods/graphene oxide core/shell nanocomposites, ACS Appl. Mater. Interfaces 5 (2013) 12911-12920. 
100. Y. Wang, K. Wang, J. Zhao, X. Liu, J. Bu, X. Yan et al., Multifunctional mesoporous silica-coated graphene nanosheet used for chemo-photothermal synergistic targeted therapy of glioma, J. Am. Chem. Soc. 135 (2013) 4799-4804.

101. Y. W. Chen, P. J. Chen, S. H. Hu, I. W. Chen and S. Y. Chen, NIR-triggered synergic photo-chemothermal therapy delivered by reduced graphene oxide/carbon/mesoporous silica nanocookies, Adv. Funct. Mater. 24 (2014) 451-459.

102. S. H. Hu, R. H. Fang, Y. W. Chen, B. J. Liao, I. W. Chen and S. Y. Chen, Photoresponsive protein-graphene-protein hybrid capsules with dual targeted heat-triggered drug delivery approach for enhanced tumor therapy, Adv. Funct. Mater. 24 (2014) $4144-4155$.

103. J. Bai, Y. Liu and X. Jiang, Multifunctional PEG-GO/CuS nanocomposites for near-infrared chemo-photothermal therapy, Biomater. 35 (2014) 5805-5813.

104. J. Chen, H. Liu, C. Zhao, G. Qin, G. Xi, T. Li et al., One-step reduction and PEGylation of graphene oxide for photothermally controlled drug delivery, Biomaterials 35 (2014) $4986-4995$.

105. H. Wan, Y. Zhang, Z. Liu, G. Xu, G. Huang, Y. Ji et al., Facile fabrication of a nearinfrared responsive nanocarrier for spatiotemporally controlled chemo-photothermal synergistic cancer therapy, Nanoscale 6 (2014) 8743-8753.

106. L. Feng, K. Li, X. Shi, M. Gao, J. Liu and Z. Liu, Smart pH-responsive nanocarriers based on nano-graphene oxide for combined chemo-and photothermal therapy overcoming drug resistance, Adv. Healthcare Mater. 3 (2014) 1261-1271.

107. Y.-J. Lu, C.-W. Lin, H.-W. Yang, K.-J. Lin, S.-P. Wey, C.-L. Sun et al., Biodistribution of PEGylated graphene oxide nanoribbons and their application in cancer chemo-photothermal therapy, Carbon 74 (2014) 83-95.

108. J.-M. Shen, F.-Y. Gao, L.-P. Guan, W. Su, Y.-J. Yang, Q.-R. Li et al., Graphene oxideFe $3 \mathrm{O} 4$ nanocomposite for combination of dual-drug chemotherapy with photothermal therapy, RSC Adv. 4 (2014) 18473-18484.

109. W. Miao, G. Shim, S. Lee, S. Lee, Y. S. Choe and Y.-K. Oh, Safety and tumor tissue accumulation of pegylated graphene oxide nanosheets for co-delivery of anticancer drug and photosensitizer, Biomaterials 34 (2013) 3402-3410.

110. L. Zhou, S. Wei, X. Ge, J. Zhou, H. Jiang, F. Li et al., Combination of chemotherapy and photodynamic therapy using graphene oxide as drug delivery system, J. Photochem. Photobiol. B, Biol. 135 (2014) 7-16.

111. F.-F. Cheng, W. Chen, L.-H. Hu, G. Chen, H.-T. Miao, C. Li et al., Highly dispersible PEGylated graphene/Au composites as gene delivery vector and potential cancer therapeutic agent, J. Mater. Chem. B 1 (2013) 4956-4962.

112. Y. Tao, E. Ju, J. Ren and X. Qu, Immunostimulatory oligonucleotides-loaded cationic graphene oxide with photothermally enhanced immunogenicity for photothermal/immune cancer therapy, Biomaterials 35 (2014) 9963-9971.

113. F. Yin, K. Hu, Y. Chen, M. Yu, D. Wang, Q. Wang et al., SiRNA Delivery with PEGylated graphene oxide nanosheets for combined photothermal and genetherapy for pancreatic cancer, Theranostics 7 (2017) 1133.

114. F. Zhi, H. Dong, X. Jia, W. Guo, H. Lu, Y. Yang et al., Functionalized graphene oxide mediated adriamycin delivery and miR-21 gene silencing to overcome tumor multidrug resistance in vitro, PloS One 8 (2013) e60034.

115. Y. Zeng, Z. Yang, H. Li, Y. Hao, C. Liu, L. Zhu et al., Multifunctional nanographene oxide for targeted gene-mediated thermochemotherapy of drug-resistant tumour, Sci. Rep. 7 (2017).

116. B. Tian, C. Wang, S. Zhang, L. Feng and Z. Liu, Photothermally enhanced photodynamic therapy delivered by nano-graphene oxide, ACS Nano 5 (2011) 7000-7009. 
117. A. Sahu, W. I. Choi, J. H. Lee and G. Tae, Graphene oxide mediated delivery of methylene blue for combined photodynamic and photothermal therapy, Biomaterials 34 (2013) 6239-6248.

118. Z. Chen, Z. Li, J. Wang, E. Ju, L. Zhou, J. Ren et al., A multi-synergistic platform for sequential irradiation-activated high-performance apoptotic cancer therapy, $A d v$. Funct. Mater. 24 (2014) 522-529.

119. B.-P. Jiang, L.-F. Hu, D.-J. Wang, S.-C. Ji, X.-C. Shen and H. Liang, Graphene loading water-soluble phthalocyanine for dual-modality photothermal/photodynamic therapy via a one-step method, J. Mater. Chem. B 2 (2014) 7141-7148.

120. J. Lin, X. Chen and P. Huang, Graphene-based nanomaterials for bioimaging, $A d v$. Drug Deliv. Rev. 105 (2016) 242-254.

121. X. Sun, Z. Liu, K. Welsher, J. T. Robinson, A. Goodwin, S. Zaric et al., Nano-graphene oxide for cellular imaging and drug delivery, Nano Res. 1 (2008) 203-212.

122. C. Peng, W. Hu, Y. Zhou, C. Fan and Q. Huang, Intracellular imaging with a graphenebased fluorescent probe, Small 6 (2010) 1686-1692.

123. Z. Sun, P. Huang, G. Tong, J. Lin, A. Jin, P. Rong et al., VEGF-loaded graphene oxide as theranostics for multi-modality imaging-monitored targeting therapeutic angiogenesis of ischemic muscle, Nanoscale 5 (2013) 6857-6866.

124. K. L. Schroeder, R. V. Goreham and T. Nann, Graphene quantum dots for theranostics and bioimaging, Pharmaceut. Res. 33 (2016) 2337-2357.

125. S. Zhu, J. Zhang, C. Qiao, S. Tang, Y. Li, W. Yuan et al., Strongly green-photoluminescent graphene quantum dots for bioimaging applications, Chem. Commun. 47 (2011) 6858-6860.

126. Q. Liu, B. Guo, Z. Rao, B. Zhang and J. R. Gong, Strong two-photon-induced fluorescence from photostable, biocompatible nitrogen-doped graphene quantum dots for cellular and deep-tissue imaging, Nano Lett. 13 (2013) 2436-2441.

127. X. Zhou, X. Huang, X. Qi, S. Wu, C. Xue, F. Y. Boey et al., In situ synthesis of metal nanoparticles on single-layer graphene oxide and reduced graphene oxide surfaces, J. Phys. Chem. C 113 (2009) 10842-10846.

128. X. Wang, P. Huang, L. Feng, M. He, S. Guo, G. Shen et al., Green controllable synthesis of silver nanomaterials on graphene oxide sheets via spontaneous reduction, $R S C A d v .2$ (2012) 3816-3822.

129. J. Huang, L. Zhang, B. Chen, N. Ji, F. Chen, Y. Zhang et al., Nanocomposites of sizecontrolled gold nanoparticles and graphene oxide: Formation and applications in SERS and catalysis, Nanoscale 2 (2010) 2733-2738.

130. Z. Liu, Z. Guo, H. Zhong, X. Qin, M. Wan and B. Yang, Graphene oxide based surfaceenhanced Raman scattering probes for cancer cell imaging, Phys. Chem. Chem. Phys. 15 (2013) 2961-2966.

131. J. Huang, C. Zong, H. Shen, M. Liu, B. Chen, B. Ren et al., Mechanism of cellular uptake of graphene oxide studied by surface-enhanced raman spectroscopy, Small 8 (2012) $2577-2584$.

132. Z.-L. Song, Z. Chen, X. Bian, L.-Y. Zhou, D. Ding, H. Liang et al., Alkyne-functionalized superstable graphitic silver nanoparticles for Raman imaging, J. Am. Chem. Soc. 136 (2014) 13558-13561.

133. A. Gizzatov, V. Keshishian, A. Guven, A. M. Dimiev, F. Qu, R. Muthupillai et al., Enhanced MRI relaxivity of aquated Gd 3+ ions by carboxyphenylated water-dispersed graphene nanoribbons, Nanoscale 6 (2014) 3059-3063.

134. W. Chen, P. Yi, Y. Zhang, L. Zhang, Z. Deng and Z. Zhang, Composites of aminodextran-coated $\mathrm{Fe} 3 \mathrm{O} 4$ nanoparticles and graphene oxide for cellular magnetic resonance imaging, ACS Appl. Mater. Interfaces 3 (2011) 4085-4091. 
135. Y. H. Hu, The first magnetic-nanoparticle-free carbon-based contrast agent of magnetic-resonance imaging-fluorinated graphene oxide, Small 10 (2014) 1451-1452.

136. H. Hong, K. Yang, Y. Zhang, J. W. Engle, L. Feng, Y. Yang et al., In vivo targeting and imaging of tumor vasculature with radiolabeled, antibody-conjugated nanographene, ACS Nano 6 (2012) 2361-2370.

137. H. Hong, Y. Zhang, J. W. Engle, T. R. Nayak, C. P. Theuer, R. J. Nickles et al., In vivo targeting and positron emission tomography imaging of tumor vasculature with $66 \mathrm{Ga}-$ labeled nano-graphene, Biomaterials 33 (2012) 4147-4156.

138. Y. Fazaeli, O. Akhavan, R. Rahighi, M. R. Aboudzadeh, E. Karimi and H. Afarideh, In vivo SPECT imaging of tumors by 198,199 Au-labeled graphene oxide nanostructures, Mater. Sci. Eng. C $4 \mathbf{5}$ (2014) 196-204.

139. B. Cornelissen, S. Able, V. Kersemans, P. A. Waghorn, S. Myhra, K. Jurkshat et al., Nanographene oxide-based radioimmunoconstructs for in vivo targeting and SPECT imaging of HER2-positive tumors, Biomaterials 34 (2013) 1146-1154.

140. C. Wang, J. Li, C. Amatore, Y. Chen, H. Jiang and X. M. Wang, Gold nanoclusters and graphene nanocomposites for drug delivery and imaging of cancer cells, Angew. Chem. Int. Edn. 50 (2011) 11644-11648.

141. M.-L. Chen, Y.-J. He, X.-W. Chen and J.-H. Wang, Quantum-dot-conjugated graphene as a probe for simultaneous cancer-targeted fluorescent imaging, tracking and monitoring drug delivery, Bioconjugate Chem. 24 (2013) 387-397.

142. C. Wang, B. Chen, M. Zou and G. Cheng, Cyclic RGD-modified chitosan/graphene oxide polymers for drug delivery and cellular imaging, Colloids Surf. B, Biointerfaces 122 (2014) 332-340.

143. X. Ma, Q. Qu, Y. Zhao, Z. Luo, Y. Zhao, K. W. Ng et al., Graphene oxide wrapped gold nanoparticles for intracellular Raman imaging and drug delivery, J. Mater. Chem. B 1 (2013) 6495-6500.

144. Y. Wang, L. Polavarapu and L. M. Liz-Marzán, Reduced graphene oxide-supported gold nanostars for improved SERS sensing and drug delivery, ACS Appl. Mater. Interfaces 6 (2014) 21798-21805.

145. M. Zhang, Y. Cao, Y. Chong, Y. Ma, H. Zhang, Z. Deng et al., Graphene oxide based theranostic platform for $\mathrm{T}$ 1-weighted magnetic resonance imaging and drug delivery, ACS Appl. Mater. Interfaces 5 (2013) 13325-13332.

146. C. Wang, S. Ravi, U. S. Garapati, M. Das, M. Howell, J. Mallela et al., Multifunctional chitosan magnetic-graphene (CMG) nanoparticles: A theranostic platform for tumortargeted co-delivery of drugs, genes and MRI contrast agents, J. Mater. Chem. B 1 (2013) 4396-4405.

147. H.-W. Yang, C.-Y. Huang, C.-W. Lin, H.-L. Liu, C.-W. Huang, S.-S. Liao et al., Gadolinium-functionalized nanographene oxide for combined drug and microRNA delivery and magnetic resonance imaging, Biomaterials 35 (2014) 6534-6542.

148. S. H. Hu, Y. W. Chen, W. T. Hung, I. W. Chen and S. Y. Chen, Quantum-dot-tagged reduced graphene oxide nanocomposites for bright fluorescence bioimaging and photothermal therapy monitored in situ, Adv. Mater. 24 (2012) 1748-1754.

149. X. Yan, G. Niu, J. Lin, A. J. Jin, H. Hu, Y. Tang et al., Enhanced fluorescence imaging guided photodynamic therapy of sinoporphyrin sodium loaded graphene oxide, Biomaterials 42 (2015) 94-102.

150. Y. Wang, H. Wang, D. Liu, S. Song, X. Wang and H. Zhang, Graphene oxide covalently grafted upconversion nanoparticles for combined NIR mediated imaging and photothermal/photodynamic cancer therapy, Biomaterials 34 (2013) 7715-7724. 
151. Y. Cho and Y. Choi, Graphene oxide-photosensitizer conjugate as a redox-responsive theranostic agent, Chem. Commun. 48 (2012) 9912-9914.

152. Y. Cho, H. Kim and Y. Choi, A graphene oxide-photosensitizer complex as an enzymeactivatable theranostic agent, Chem. Commun. 49 (2013) 1202-1204.

153. O. Akhavan and E. Ghaderi, Graphene nanomesh promises extremely efficient in vivo photothermal therapy, Small 9 (2013) 3593-3601.

154. J. L. Li, H. C. Bao, X. L. Hou, L. Sun, X. G. Wang and M. Gu, Graphene oxide nanoparticles as a nonbleaching optical probe for two-photon luminescence imaging and cell therapy, Angew. Chem. Int. Edn. 51 (2012) 1830-1834.

155. Y. K. Kim, H. K. Na, S. Kim, H. Jang, S. J. Chang and D. H. Min, One-pot synthesis of multifunctional Au@ graphene oxide nanocolloid Core@ shell nanoparticles for raman bioimaging, Photothermal and Photodynamic Therapy, Small 11 (2015) $2527-2535$.

156. S. Wang, Q. Zhang, X. F. Luo, J. Li, H. He, F. Yang et al., Magnetic graphene-based nanotheranostic agent for dual-modality mapping guided photothermal therapy in regional lymph nodal metastasis of pancreatic cancer, Biomaterials 35 (2014) 9473-9483.

157. Y.-W. Wang, Y.-Y. Fu, Q. Peng, S.-S. Guo, G. Liu, J. Li et al., Dye-enhanced graphene oxide for photothermal therapy and photoacoustic imaging, J. Mater. Chem. B 1 (2013) $5762-5767$.

158. Z. Sheng, L. Song, J. Zheng, D. Hu, M. He, M. Zheng et al., Protein-assisted fabrication of nano-reduced graphene oxide for combined in vivo photoacoustic imaging and photothermal therapy, Biomaterials 34 (2013) 5236-5243.

159. H. Zhang, H. Wu, J. Wang, Y. Yang, D. Wu, Y. Zhang et al., Graphene oxide-BaGdF 5 nanocomposites for multi-modal imaging and photothermal therapy, Biomaterials 42 (2015) 66-77.

160. X. Shi, H. Gong, Y. Li, C. Wang, L. Cheng and Z. Liu, Graphene-based magnetic plasmonic nanocomposite for dual bioimaging and photothermal therapy, Biomaterials 34 (2013) 4786-4793.

161. K. Yang, L. Hu, X. Ma, S. Ye, L. Cheng, X. Shi et al., Multimodal imaging guided photothermal therapy using functionalized graphene nanosheets anchored with magnetic nanoparticles, Adv. Mater. 24 (2012) 1868-1872.

162. Y. Jin, J. Wang, H. Ke, S. Wang and Z. Dai, Graphene oxide modified PLA microcapsules containing gold nanoparticles for ultrasonic/CT bimodal imaging guided photothermal tumor therapy, Biomaterials 34 (2013) 4794-4802.

163. P. Rong, K. Yang, A. Srivastan, D. O. Kiesewetter, X. Yue, F. Wang et al., Photosensitizer loaded nano-graphene for multimodality imaging guided tumor photodynamic therapy, Theranostics 4 (2014) 229.

164. X. Yan, H. Hu, J. Lin, A. J. Jin, G. Niu, S. Zhang et al., Optical and photoacoustic dual-modality imaging guided synergistic photodynamic/photothermal therapies, Nanoscale 7 (2015) 2520-2526.

165. G. Gollavelli and Y.-C. Ling, Magnetic and fluorescent graphene for dual modal imaging and single light induced photothermal and photodynamic therapy of cancer cells, Biomaterials 35 (2014) 4499-4507.

166. X. Bian, Z.-L. Song, Y. Qian, W. Gao, Z.-Q. Cheng, L. Chen et al., Fabrication of graphene-isolated-Au-nanocrystal nanostructures for multimodal cell imaging and photothermal-enhanced chemotherapy, Sci. Rep. 4 (2014).

167. J. Shi, L. Wang, J. Zhang, R. Ma, J. Gao, Y. Liu et al., A tumor-targeting near-infrared laser-triggered drug delivery system based on GO@ Ag nanoparticles for chemo-photothermal therapy and X-ray imaging, Biomaterials 35 (2014) 5847-5861. 
168. Y. Wang, R. Huang, G. Liang, Z. Zhang, P. Zhang, S. Yu et al., MRI-visualized, dualtargeting, combined tumor therapy using magnetic graphene-based mesoporous silica, Small 10 (2014) 109-116.

169. S. P. Sherlock, S. M. Tabakman, L. Xie and H. Dai, Photothermally enhanced drug delivery by ultrasmall multifunctional $\mathrm{FeCo} /$ graphitic shell nanocrystals, ACS Nano 5 (2011) 1505-1512. 